Sonderdruck aus

Thomas Heinemann / Hans-Georg Dederer / Tobias Cantz (Hg.)

\title{
Entwicklungsbiologische Totipotenz in Ethik und Recht
}

Zur normativen Bewertung von totipotenten menschlichen Zellen

Mit 3 Abbildungen

V\& R unipress

ISBN 978-3-8471-0342-4

ISBN 978-3-8470-0342-7 (E-Book) 


\section{Ein bisschen Embryo? Begriffliche, ontologische und normative Überlegungen zur totipotenzbasierten Legaldefinition von $\gg$ Embryo«}

\section{Einleitung}

»Ein bisschen schwanger gibt es nicht«, weiß der Volksmund. Entwicklungsbiologen und Fortpflanzungsmedizinern kommt dieser Gemeinplatz nicht mehr so leicht über die Lippen. Je intensiver man sich den biologischen Details der Befruchtungs- und Einnistungsphase zuwendet, desto zweifelhafter erscheint der Versuch, den Beginn einer Schwangerschaft exakt und auf nichtwillkürliche Weise zu datieren. Betrachtet man zusätzlich die technischen Möglichkeiten der extrakorporalen Erzeugung entwicklungsfähiger Embryonen aus totipotenten Zellen, so scheint neben »ein bisschen schwanger« auch »ein bisschen Embryo" durchaus im Reiche der Natur zu sein.

Im deutschen Embryonenschutz- und im Stammzellgesetz sind zwei Rechtsbegriffe von "Embryo « definiert worden, die sich auf die Zelleigenschaft der Totipotenz stützen und dieser damit eine ontologische und normative Bedeutung beimessen, die angesichts der vielfältigen divergierenden Intuitionen und Argumente zur sogenannten Statusfrage nicht leicht zu rechtfertigen ist. Der vorliegende Beitrag diskutiert die Schwierigkeiten, den ontologischen, moralischen und rechtlichen Status totipotenter Humanzellen plausibel zu begründen, und argumentiert insbesondere, dass zwischen Grundannahmen der Substanzontologie und naturphilosophischen Kontinuitätsüberlegungen unaufhebbare Spannungen bestehen, die der Gesetzgeber durch seine Legaldefinitionen einseitig gelöst hat, ohne über hinreichend belastbare Argumente zu verfügen.

Die Suche nach nichtwillkürlichen Einschnitten in einem entwicklungsbiologischen Kontinuum ist eine große Herausforderung für die Medizinethik, die sich im strafrechtlichen Kontext noch verschärft. Der Gesetzgeber kann die Frage nicht auf sich beruhen lassen, wann genau ein Embryo zu existieren beginnt und was überhaupt als ein Embryo zählt. Um ein strafbewehrtes Verbot des Klonens und der verbrauchenden Embryonenforschung erlassen zu können, musste er den unscharf gewordenen Embryonenbegriff in eine Legaldefinition überführen, die dem Bestimmtheitsgebot des Strafrechts Rechnung trägt. 
Neue Komplikationen und möglicherweise zusätzlicher Regelungsbedarf sind durch die seit 2006 bekannte und seither fortentwickelte Technik der Reprogrammierung differenzierter Körperzellen zu iPS-Zellen (induzierten pluripotenten Stammzellen) entstanden. Da sich die therapeutischen Hoffnungen Regeneration von Geweben und Organen, Gentherapie von Erbkrankheiten, Immuntherapie von Krebs - primär auf pluripotente Zellen richten, nicht auf totipotente, war die Erleichterung zunächst groß, dass die Gewinnbarkeit von iPS-Zellen durch Reprogrammierungstechniken den Rückgriff auf embryonale Stammzellen überflüssig macht. Mittlerweile wird es als möglich angesehen, dass die somatischen Ausgangszellen im Zuge der Reprogrammierung ein Stadium der Totipotenz erreichen oder transient durchlaufen. ${ }^{1}$ Diese Vermutung wirft die Fragen auf, ob derartige Zellen von der totipotenzbasierten Embryodefinition des Stammzellgesetzes und von dessen Verbotsnormen erfasst sind und, falls nicht, ob darin eine zu schließende Schutzlücke zu sehen ist. ${ }^{2}$

Da die epistemischen Unsicherheiten hinsichtlich der Totipotenz reprogrammierter Körperzellen groß sind, ist die Debatte einstweilen auf Gedankenexperimente angewiesen. ${ }^{3}$ Menschliche Stammzellen weisen einen hochspezialisierten Phänotyp auf. Sie sind zur Entwicklung auf spezielle Umgebungsbedingungen angewiesen und tragen in utero durch hormonelle Interaktion selbst zur Herstellung dieser Bedingungen bei. Ob in Zellkulturen gehaltene Zellen eines totipotenten Phänotyps tatsächlich die Fähigkeit besitzen, sich bei Vorliegen weiterer Voraussetzungen zu teilen und zu einem Menschen $\mathrm{zu}$ entwickeln, ist keineswegs ausgemacht. ${ }^{4}$ Ein »Totipotenzmarker « ist bisher nicht gefunden worden.

Ontologisch ist Totipotenz eine Dispositionseigenschaft, also eine solche, die über das Verhalten definiert ist, das die Trägersubstanz bei Vorliegen geeigneter Manifestationsbedingungen zeigen würde. Die geeigneten Bedingungen experimentell herzustellen verbietet sich bei reprogrammierten Humanzellen aber, da dies einem ethisch inakzeptablem Menschenversuch und im Erfolgsfall einem Klonieren gleichkäme. Pointiert: Wenn die fraglichen Zellen totipotent

1 Zu den entwicklungs- und zellbiologischen Einzelheiten vgl. die Beiträge von Cantz, Ott und Sgodda in diesem Band, $13-77$.

2 Von der Embryodefinition des Embryonenschutzgesetzes sind sie nicht erfasst, weil reprogrammierte Körperzellen nicht »einem Embryo entnommen« wurden (ESchG, § 8, Abs. 1). Vgl. dazu Günther/Kaiser/Taupitz 2008, § 8 Rn. 62.

3 Siehe das im Rahmen des BMBF-geförderten Projekts "Entwicklungsbiologische Totipotenz« entwickelte Gedankenexperiment der »modifizierten Reprogrammierung «, in dem durch eine Fortentwicklung der Yamanaka-Methode differenzierte Körperzellen in das Stadium der Totipotenz überführt und in vitro kultiviert werden, so dass sich aus ihnen pluripotente Stammzellen für die biomedizinische Forschung und Therapie gewinnen lassen. Vgl. AdvenaRegnery et al. 2012, 220-221.

$4 \mathrm{Vgl}$. Ott in diesem Band, $72 \mathrm{f}$. 
sind, dann ist durch das Stammzellgesetz strafbewehrt verboten, sie experimentell daraufhin zu untersuchen, $o b$ sie es sind, ob also ihre Erzeugung und Verwendung das Stammzellgesetz verletzt. Angesichts dieser vertrackten Beweissituation ist es nicht verwunderlich, dass bis dato kein einziges Strafverfahren wegen eines Verstoßes gegen das Stammzellgesetz zur Hauptverhandlung gelangt ist. Auch bei vermuteten Verstößen gegen Bestimmungen des Embryonenschutzgesetzes kommt ohne einen objektiven Totipotenznachweis allenfalls die Strafbarkeit wegen Versuchs in Betracht. ${ }^{5}$

\section{Legaldefinitionen von »Embryo«}

Der deutsche Gesetzgeber hat »Embryo« im Strafrecht gleich zweimal definiert. Im Embryonenschutzgesetz von 1990 heißt es:

"Als Embryo im Sinne dieses Gesetzes gilt bereits die befruchtete, entwicklungsfähige menschliche Eizelle vom Zeitpunkt der Kernverschmelzung an, ferner jede einem Embryo entnommene totipotente Zelle, die sich bei Vorliegen der dafür erforderlichen weiteren Voraussetzungen zu teilen und zu einem Individuum zu entwickeln vermag «. ${ }^{6}$

Im 2002 verabschiedeten Stammzellgesetz, das die Einfuhr und Verwendung embryonaler menschlicher Stammzellen regelt, gilt »bereits jede menschliche totipotente Zelle« als Embryo, also auch die nicht durch Befruchtung entstandene. ${ }^{7} \mathrm{Da}$ das ESchG seinerzeit nicht geändert wurde, koexistieren seither zwei voneinander abweichende Rechtsbegriffe von "Embryo«.

Eine weite Legaldefinition verwendet auch der Europäische Gerichtshof, der 2011 im Verfahren Brüstle gegen Greenpeace, das die Patentierbarkeit von aus menschlichen embryonalen Stammzellen gewonnenen Zellen zum Gegenstand hatte, entschied, "dass der Begriff des menschlichen Embryos [...] weit auszulegen ist " und "jede unbefruchtete menschliche Eizelle, in die ein Zellkern aus einer ausgereiften menschlichen Zelle transplantiert worden ist oder die durch Parthenogenese zur Teilung und Weiterentwicklung angeregt worden ist $~^{8}$, umfasst. Die Begründung lautete, dass diese Entitäten - der EuGH spricht unkorrekt von "Organismen " ${ }^{9}$ - »infolge der zu ihrer Gewinnung verwendeten

5 Vgl. Günther/Kaiser/Taupitz 2008, $\$ 2$ Rn. 38.

6 ESchG, $\$ 8$, Abs. 1. Die Bezeichnung »Kernverschmelzung« entspricht nicht mehr dem Stand der entwicklungsbiologischen Forschung, denn die beiden Vorkerne verschmelzen bei Säugern nicht zu einem, sondern teilen sich unmittelbar nach dem Kontakt, wobei sich beide Kernmembranen auflösen und die Chromosomen sich verdoppeln. Vgl. dazu Günther/Kaiser/ Taupitz 2008, §8 Rn. 28.

7 StZG, $\$ 3$, Nr. 4.

8 Urteil des EuGH vom 18.10.2011 - C34/10.

9 Als »Organismus« gilt biologisch erst »ein koordiniertes und zur Selbstorganisation und 
Technik geeignet [sind], wie der durch Befruchtung einer Eizelle entstandene Embryo den Prozess der Entwicklung eines Menschen in Gang zu setzen «. ${ }^{10}$ Der EuGH argumentiert also wie schon das deutsche Stammzellgesetz mit einer funktionalen Äquivalenz: Was einem durch Befruchtung entstandenen Embryo hinsichtlich der Entwicklungsfähigkeit funktional äquivalent ist, sei selbst ein Embryo. Eine ätiologische Äquivalenz sei nicht erforderlich. ${ }^{11}$

\section{Normative Spannungen und das Potentialitätsargument}

Durch das ESchG und das StZG sind normative Spannungen zum Gesetz über den Schwangerschaftsabbruch entstanden, die man so zusammenfassen kann, dass im deutschen Recht extrakorporale totipotente Zellen durch Strafgesetze stärker geschützt sind als ein Fötus im Mutterleib, der straflos getötet werden darf.

Wie spannungsvoll diese Rechtslage in normativer Hinsicht ist, wird besonders deutlich, wenn man sie in den Kontext des medizinethischen Potentialitätsarguments stellt, dem das ESchG und das StZG verpflichtet zu sein scheinen, wenn sie den Schutzstatus der totipotenten Zelle aus dem Entwicklungspotential ableiten. Das Potentialitätsargument besagt, dass einem Embryo moralisch relevante Eigenschaften, Menschenwürde oder Rechte eines geborenen Menschen zukommen, weil er das Potential hat, sich zu einem solchen zu entwickeln, oder, kürzer und unklarer: weil er potentiell ein Mensch ist. ${ }^{12}$ Der Überlegung, dass Entwicklungsfähigkeit zu einem Ganzen als solche für den Schutzstatus relevant ist, folgt offenbar auch der Gesetzgeber: Eine totipotente Humanzelle ist

Selbststeuerung fähiges Ganzes, das sich deutlich von seinen Teilen unterscheiden lässt«. Da nun »die ersten Differenzierungsschritte des Zellverbands unabdingbar der Ablesung wichtiger Informationen aus dem Genom bedürfen, die aber in den ersten Tagen noch nicht aus dem autonomen, neuen Genom gesteuert werden, sondern durch Wachstumsfaktoren reguliert werden, die ausschließlich vom Zellsaft der mütterlichen Eizelle stammen«, ist eine totipotente Humanzelle im biologischen Sinn kein Organismus. Günther/Kaiser/Taupitz 2008, \$ 8 Rn. 30 mit Verweis auf Reich 2004, 117-118.

10 Urteil des EuGH vom 18.10.2011 - C34/10.

11 Die vom EuGH postulierte funktionale Äquivalenz ist vorsichtigerweise auf das »Ingangsetzen « einer Entwicklung beschränkt, was die Interpretation zulässt, dass das Zutreffen der Embryodefinition vom tatsächlichen biologischen Entwicklungspotential der fraglichen technisch erzeugten Entitäten unabhängig ist. Zellkerntransferexperimente haben schon in den 1980er Jahren ergeben, »dass Säugerkeime, deren Genom sich nur von einem Elternteil herleitet, nicht entwicklungsfähig sind " (Huber 2009, 78, vgl. 69-83). Bis heute ist die Parthenogenese eines voll entwickelten Lebewesens aus unbefruchteten Eizellen bei höheren Säugetieren nicht nachgewiesen, wofür die Unerlässlichkeit der geschlechtsspezifischen Methylierung (genomisches Imprinting) verantwortlich gemacht wird.

12 Vgl. Damschen/Schönecker (Hg.) 2003, 5-6. 
schutzwürdig, weil sie sich »bei Vorliegen der dafür erforderlichen weiteren Voraussetzungen $\mathrm{zu}$ teilen und $\mathrm{zu}$ einem Individuum $\mathrm{zu}$ entwickeln vermag«. Irritierend ist nun der Umstand, dass im ESchG und im StZG Totipotenz gerade nicht einen Anspruch auf Unterstützung der Weiterentwicklung begründet, denn eine Weiterentwicklung in vitro oder in vivo wäre ein Klonen, das das ESchG (\$6) gerade unter Strafe stellt. Beide Gesetze scheinen zwar auf der Überlegung zu beruhen, dass die Schutzwürdigkeit des Embryos sich aus seiner Fähigkeit ergibt, sich zu einem Individuum zu entwickeln. Diese Begründung des Schutzstatus entspricht dem Potentialitätsargument, doch erhält die Entwicklungsfähigkeit hier eine gegenläufige Funktion zu der, die sie in der Debatte über die Zulässigkeit des Schwangerschaftsabbruchs hat: Aus der Sicht des Potentialitätsarguments ist es irritierend, dass der Gesetzgeber zwar erlaubt, entwicklungsfähige Entitäten zum Zweck der Fortpflanzung zu erzeugen, zugleich aber den widerrechtlich erzeugten den Transfer in einen Uterus verweigert und sie damit de facto dem Tod weiht. Dies monieren auch die Kommentatoren des ESchG: »Wenn man das Transferverbot für verfassungsgemäß und für sachgerecht hält, wäre es folgerichtiger, der erzeugten entwicklungsfähigen Entität gar nicht erst den Status eines Embryos zuzusprechen, den man ihm doch sogleich wieder zu nehmen gehalten ist. ${ }^{13}$

Heike Baranzke erklärt die normative Spannung so, dass Totipotenz im ESchG und im StZG als »Problemindikator « fungiere: Solche Zellen dürfen nicht nur nicht in den Uterus eingesetzt, sondern gar nicht erst isoliert oder erzeugt werden. Es sei insofern "ersichtlich, dass die rechtliche Rezeption des Totipotenzbegriffs diesen nicht normativ als Begründung für einen Anspruch auf Weiterentwicklung, sondern rein deskriptiv verwendet «. ${ }^{14}$ Aus der Perspektive einer totipotenzbasierten Embryodefinition schützt allein ein strafbewehrtes Verbot der Erzeugung totipotenter Humanzellen davor, in die Situation moralisch und verfassungsrechtlich unhaltbarer Abwägungen zu geraten. Es ist in diesem Sinne vorgeschlagen worden, bei der Reprogrammierung menschlicher Zellen durch Eingriffe in die Ausgangszellen Totipotenz von vornherein zu verhindern.

\section{Konfligierende Anforderungen und Intuitionen}

Der Gesetzgeber ist bereits im Jahr 2002 von der Enquete-Kommission »Recht und Ethik in der Medizin « und 2003 vom Nationalen Ethikrat dazu aufgefordert worden, das ESchG in ein umfassendes Fortpflanzungsmedizingesetz zu über-

13 Günther/Kaiser/Taupitz 2008, § 8 Rn. 13.

14 Baranzke in diesem Band, 191. 
führen, das die verstreuten Regelungen zusammenführt, die betroffenen Grundrechtspositionen in einen angemessenen Ausgleich bringt und die oft beschriebenen Wertungswidersprüche ${ }^{15}$ beseitigt. Ein entsprechendes internes Eckpunktepapier des Bundesgesundheitsministeriums wurde aber nicht weiter verfolgt. $^{16}$

Der Gesetzgeber tut sich mit einer solchen umfassenden Initiative so schwer, weil er sich wie der Medizinethiker der Aufgabe gegenübersieht, einer Reihe von heterogenen Intuitionen und Annahmen Rechnung zu tragen, die in ein spannungsvolles Anforderungsprofil münden. Zum einen hat der Gesetzgeber in das ESchG und das StZG ontologische Annahmen eingebaut, die über entwicklungsbiologisches Wissen hinausgehen, nämlich solche der aristotelischen Substanzontologie. Diesen Annahmen stehen starke Intuitionen über den kontinuierlichen Charakter biologischer Entwicklungen gegenüber. Weiterhin scheint die Gesetzgebung bemüht, einer Reihe von heterogenen moralischen Intuitionen Rechnung zu tragen. Im Einzelnen:

- Es gibt eine starke moralische Intuition hinsichtlich der Ächtung des Klonens. Die Natur kann etwas, was Menschen im Labor nicht dürfen bzw. sich mit guten Gründen nicht erlauben sollten, nämlich genetisch identische menschliche Zwillinge erzeugen.

- Es gibt eine allgemeinere und etwas schwächere Intuition, dass im Bereich der menschlichen Fortpflanzung "Natürlichkeit « etwas Gutes ist, so dass es stets begründungsbedürftig ist, in Entwicklungsvorgänge technisch einzugreifen oder sie in vitro zu induzieren, während es nicht begründungsbedürftig ist, natürlichen Entwicklungen ihren Lauf zu lassen. Die moralische Privilegierung natürlicher Verläufe ist notorisch schwierig zu präzisieren, aber sie scheint unter anderem wichtig, um zu erklären, warum die beträchtliche natürliche Abortrate zwischen Befruchtung und Einnistung - bis zu 50 \% der befruchteten Eizellen gehen spontan zugrunde - auch aus Sicht der Vertreter des uneingeschränkten Lebensschutzes kein gravierendes ethisches Problem darstellt.

- Ferner ist weithin unstrittig, dass alle Menschen unabhängig davon, welche technischen Eingriffe an ihrer Genese beteiligt waren, Menschenwürde be-

15 Vgl. dazu Günther/Kaiser/Taupitz 2008, Einf. B 57, 59, 60. Dietrich und Czerner fassen diese Spannungen so zusammen, dass der strafrechtlichen Regelung des Schwangerschaftsabbruchs mit ihren »teilweise erheblich auseinanderfallenden zeitlichen Zäsuren« ein »System des abgestuften Lebensschutzes i. S. v. Art. 2 Abs. 2 S. 1 GG - nicht des Würdeschutzes nach Art. 1 Abs. 1 GG« zugrunde liegt, während diese »für das Kernstrafrecht prägende Wertentscheidung eine gewisse Umkehrung [erfährt], sofern es sich nicht um den Schwangerschaftsabbruch, sondern um den Schutz von Embryonen handelt«. Dietrich/Czerner 2013, 517.

16 Vgl. Günther/Kaiser/Taupitz 2008, Einf. B Rn. 10. 
sitzen und gleiche Achtung verdienen. Falls dereinst aus einem verbotenerweise durch Reprogrammierungstechniken oder durch noch exotischere Techniken der tetraploiden Komplementierung erzeugten Klon einmal ein Mensch geboren würde, so ist es geboten, ihn als unseresgleichen und als Träger von Menschenwürde anzuerkennen. ${ }^{17}$

- Die dem Potentialitätsargument zugrunde liegende, aber nicht allgemein geteilte Intuition besagt, dass Entwicklungsfähigkeit $\mathrm{zu}$ einem Ganzen als solche moralisch relevant ist. Eine totipotente Zelle wird als schutzwürdiger Embryo angesehen, weil angenommen wird, dass bei unter günstigen Bedingungen stattfindender kontinuierlicher Entwicklung, in der es keine weiteren moralisch relevanten Einschnitte gebe, ein Individuum entsteht, das Träger von Grundrechten ist. Wenn etwas die Potenz hat, sich zu einem menschlichen Individuum zu entwickeln (Potentialitätsargument) und/oder in einer kontinuierlichen Entwicklungslinie zu ihm steht (Kontinuumsargument), sei es moralisch und rechtlich mit ihm statusgleich. In der rechtsethischen Diskussion über die Zulässigkeit des Schwangerschaftsabbruchs entspricht dieser Gedanke der Figur der Vorwirkung von Grundrechten ${ }^{18}$ mit dem Unterschied, dass nun über die pränatale Vorwirkung hinaus eine pränidative angenommen wird. Dass neben Embryonen und Föten nun auch pränidative Entitäten, die erst via Legaldefinition zu Embryonen erklärt werden, Grundrechtsträger sein sollen, dürfte der strittigste Punkt der Debatte über das Totipotenzkriterium sein.

- Das oft nicht eigens ausgewiesene ontologische Fundament der SKIP-Argumente $^{19}$ wie auch des ESchG und des StZG bilden einige Grundannahmen der aristotelischen Substanzontologie: Menschen sind plötzlich in Existenz tretende, persistierende, sortal bestimmte Einzelsubstanzen; das Phasensortal "Embryo" bezieht sich auf eine frühe Phase solcher Substanzen (siehe Abschnitt 5.)

- Diese substanzontologischen Annahmen stehen in Spannung zum natur-

$17 »$ Der Vorwurf `menschenunwürdig` bezieht sich dabei nicht auf die Existenz des später geborenen Menschen, sondern auf das zu seiner Erzeugung führende Verfahren [...].» Günther/Kaiser/Taupitz 2008, \$ 6 Rn. 4.

18 Das Bundesverfassungsgericht begründet in seinem ersten Urteil zum Schwangerschaftsabbruch die Partizipation des »sich entwickelnden Leben[s]« an der Menschenwürde mithilfe eines Potentialitätsarguments: "das sich entwickelnde Leben nimmt auch an dem Schutz teil, den Art. 1 Abs. 1 GG der Menschenwürde gewährt. [...]. Die von Anfang an im menschlichen Sein angelegten potentiellen Fähigkeiten genügen, um die Menschenwürde zu begründen." Bundesverfassungsgericht 1975, 41. - Im zweiten Abtreibungsurteil heißt es: "Menschenwürde kommt schon dem ungeborenen menschlichen Leben zu.» Bundesverfassungsgericht 1993, 203 (Leitsatz 1).

19 Unter dem Akronym »SKIP« werden seit Damschen/Schönecker 2003 das Spezies-, das Kontinuums-, das Identitäts- und das Potentialitätsargument zusammengefasst. Für eine abgewogene Einschätzung der SKIP-Argumente vgl. Dietrich/Czerner 2013, 491-524. 
philosophischen Kontinuitätsprinzip, dem zufolge natürliche Entwicklungen nicht sprunghaft, sondern kontinuierlich verlaufen. Es ist begründungsbedürftig, warum die Entstehung von Lebewesen von diesem immens plausiblen Prinzip ausgenommen sein sollte (siehe Abschnitte 6. und 7.).

- Auf das Kontinuitätsprinzip stützen sich gradualistische moralische Intuitionen, denen zufolge der moralische Status und die Rechte eines werdenden Menschen mit seiner Entwicklung »mitwachsen« (siehe Abschnitt 8.).

\section{Paradoxien der Substanzontologie}

Das Potentialitätsargument kann die ihm zugeschriebene normative Kraft nicht allein aus dem Faktum der Entwicklungsfähigkeit beziehen. Dies räumen auch die meisten seiner Verfechter ein und vertreten das Argument gemeinsam mit der Annahme, dass der Embryo schon in seinen frühesten Phasen ein menschliches Wesen ist - also, in der Terminologie der SKIP-Argumente, gemeinsam mit dem Spezies- und/oder dem Identitätsargument. So werden in der Rede von "pränidativen menschlichen Entitäten ${ }^{20}$ die fraglichen ein- und mehrzelligen Gebilde schon zur sortal bestimmten Einzelsubstanz "Mensch" gezählt. Die entsprechende Argumentationsfigur lautet, dass das, was sich zu einem Individuum der menschlichen Spezies entwickelt oder entwickeln kann, schon ein Mensch ist. Paradox ausgedrückt: Etwas wird zu dem, was es schon ist, nämlich ein Mensch. Diese paradoxe Figur, die sich schon beim Kirchenvater Tertullian findet (»Ein Mensch ist auch schon, wer es wird $\aleph^{21}$ ), liegt auch der Frage "Wann ist der Mensch ein Mensch?« zugrunde, die wörtlich verstanden nur die Antwort »immer" zulässt. In der Instruktion Donum Vitae der römischen Glaubenskongregation heißt es: "Von dem Augenblick an, in dem die Eizelle befruchtet wird, beginnt ein neues Leben [...], das sich eigenständig entwickelt. Es würde niemals menschlich werden, wenn es das nicht schon von diesem Augenblick an gewesen wäre. $\aleph^{22}$ Während die Glaubenskongregation ausdrücklich die Befruchtung als Beginn eines neuen Lebewesens auszeichnet, lässt das deutsche Stammzellgesetz diese Restriktion fallen und ist insofern päpstlicher als der Papst.

Die paradoxe Figur, dass etwas zu dem wird, was es schon ist, ist freilich kein

20 Baranzke in diesem Band, 181.

21 »Uns aber, denen Mord ein für allemal verboten ist, ist es auch nicht erlaubt, die Leibesfrucht im Mutterleib [...] zu vernichten. [...] Ein Mensch ist auch schon, wer es wird [Homo est et qui est futurus]; auch jede Frucht ist schon im Samen enthalten.« Tertullian, Apologeticum, 166 (Kap. 9, 8).

22 Kongregation für die Glaubenslehre 1987, I.1. Die Kongregation zitiert hier aus ihrer früheren Erklärung zur vorsätzlichen Abtreibung, 1974, 738 (12 - 13). 
biologischer Befund. Sie erfordert die aristotelische Auffassung, dass es bei Einzelsubstanzen keine Abstufungen gibt. In der Kategorienschrift heißt es: »Die Substanz läßt also kein Mehr oder Minder zu«. ${ }^{23}$ In dynamischer Hinsicht impliziert die Nichtabstufbarkeit, dass substanzielle Veränderungen, also das Entstehen und Vergehen von Substanzen, plötzlich geschehen, im Unterschied zu akzidentellen Eigenschaftsveränderungen an einer Substanz, die Zeit brauchen. In der Kategorienschrift behandelt Aristoteles nur die »statische» Lesart des Prinzips, derzufolge »die Substanz das, was sie wesenhaft ist, nicht mehr oder minder sein kann ${ }^{24}{ }^{24}$ Wenn eine Substanz ein Mensch ist, mag dieser Mensch mehr oder weniger schön sein, mehr oder weniger weiß etc., aber er kann nicht mehr oder weniger Mensch sein. Deshalb ist auch der eine Mensch »nicht mehr Mensch als der andere ${ }^{25}{ }^{25} \mathrm{Ob}$ sich aus diesem statischen Nichtabstufbarkeitsprinzip unmittelbar die Punktförmigkeit des Entstehens und Vergehens von Substanzen folgern lässt und wie sich das Prinzip zur von Aristoteles vertretenen These der Sukzessivbeseelung des Menschen (s.u.) verhält, ist umstritten.

Relevant für unseren Zusammenhang ist der Umstand, dass verschiedene Grundannahmen der aristotelischen Substanzontologie in die Grammatik vieler natürlicher Sprachen eingebaut sind, und zwar auch dort, wo dies zu absurden Konsequenzen führt. Akzidentellen Veränderungen liegt nach Aristoteles stets ein beharrendes hypokeimenon zugrunde. Nun ist sogar bei substanziellen Veränderungen das grammatische Subjekt der Aussage meist dasjenige, was durch die Veränderung erst entsteht oder vergeht. Sagt man beispielsweise "Sokrates ist tot", so prädiziert man etwas von einer Substanz, die zu diesem Zeitpunkt nach Aristoteles' Auffassung nicht mehr existiert. ${ }^{26}$ Das ist paradox, und weil es eine in die Grammatik eingebaute Paradoxie ist, ist bei der argumentativen Verwendung der betreffenden sprachlichen Befunde große Vorsicht geboten. Wir scheinen von Sokrates zu prädizieren, dass eine bestimmte Veränderung an oder mit ihm stattgefunden hat. Aus dem sprachlichen Befund, dass das grammatische Subjekt der Aussage überdauert, zu schließen, dass auch die bezeichnete Substanz überdauert, wäre ein Fehlschluss aus der Grammatik; schließlich kann ein Mensch nicht seinen eigenen Tod überleben.

Die Verlegenheit, im Falle substanzieller Veränderungen angemessene Träger der veränderungsbezeichnenden Prädikate zu finden, hängt mit dem Umstand zusammen, dass die aristotelische Ontologie nur sortal bestimmte Substanzen

23 Aristoteles, Kategorien V, 4a.

24 Ebd.

25 Ebd.

26 »Falsch ist es z. B., wenn ein toter Mensch ein Mensch sein soll«, weil hier »in dem in die Aussage Aufgenommenen ein Gegensatz liegt, dem logisch ein Widerspruch folgt « (De Interpretatione 11, 21a), nämlich dass etwas zugleich ein Mensch und nicht ein Mensch sei. 
kennt. Jede Einzelsubstanz exemplifiziert für Aristoteles eine wohlbestimmte natürliche Art. Der Ausdruck "Embryo" gilt als ein Phasensortal, d.h. er bezeichnet eine bestimmte Phase einer bereits sortal bestimmten Substanz. Für dasjenige totipotente Gebilde, das der eigentlichen Embryonalphase vorangeht, hält die deutsche Gemeinsprache keinen eigenen Substanzausdruck bereit. In der Entwicklungsbiologie heißt das Ergebnis der Zellkernverschmelzung zunächst »Zygote«, dann »Morula«, dann »Blastozyste«. Embryonenschutz- und Stammzellgesetz vermeiden diese Ausdrücke und sprechen unterschiedslos vom »Embryo«.

Einen weiteren Fallstrick birgt die so suggestive wie undurchsichtige Semantik von »Entwicklung«. In der paradoxen Figur, dass sich etwas zu dem entwickelt, was es schon ist, wird die sprachliche Verlegenheit ausgebeutet, dass bei substanziellen Veränderungen oft kein vom terminus ad quem verschiedener terminus a quo zur Verfügung steht. So wird im Kompositum »Embryonalentwicklung" nicht unterschieden, woraus und wozu sich die fragliche Entität entwickelt, so dass der Ausdruck die Auffassung nahelegt, dass schon am Anfangspunkt dieser Entwicklung ein Embryo steht. ${ }^{27}$

"Entwicklung" ist eine äußerst suggestive und kaum noch als solche erkennbare Metapher: Wenn sich etwas ent-wickelt, wird etwas zuvor Eingewickeltes zu etwas Ausgewickeltem. Anschauliche Beispiele bieten Zeitrafferaufnahmen des Wachstums einer Pflanze. Wenn ein Keimling ein zunächst eingerolltes und sich dann entfaltendes Blatt hervortreibt, scheint das Ergebnis dieser Entwicklung die ganze Zeit schon in verborgener Form vorhanden gewesen zu sein. Diese Auffassung entspricht der beispielsweise von Anaxagoras vertretenen Präformationslehre, derzufolge ein Lebewesen schon vollständig im Keim vorgebildet ist und sich fortan nur noch entfaltet und an Größe zunimmt. Tertullian führt den vage beschriebenen Umstand, dass »jede Frucht [...] schon im Samen enthalten« sei, umstandslos zum Beleg seiner These »Homo est et qui est futurus" an (s. o.). In der Instruktion Donum Vitae heißt es, die moderne Genetik bestätige »in eindrucksvoller Weise $[. .$.$] diesen Sachverhalt, der immer$ eindeutig war", dass nämlich »das konkrete menschliche Individuum $[\ldots]$ mit all seinen genau umschriebenen charakteristischen Merkmalen" schon "vom ersten Augenblick an« in der befruchteten Eizelle enthalten sei. ${ }^{28}$ Dies mag für die Kirchenväter immer eindeutig gewesen sein, ist aber, wie wir heute wissen, empirisch falsch. Die präformistische Lehre vom Enthaltensein der Vollform im

27 Das Bundesverfassungsgericht $(1975,41)$ verwendet »sich entwickelndes Leben« und "existierendes menschliches Leben« koextensiv.

28 Glaubenskongregation 1987, I.1. 
Keim verkennt die wesentliche Rolle, die epigenetische Prozesse in der Embryonalentwicklung spielen. ${ }^{29}$

Weiterhin ist "sich entwickeln" ein reflexives Verb. Als solches impliziert es, dass die sich entwickelnde Substanz ein Agens einer an sich selbst vollzogenen Aktivität ist. Was aber Agens einer fortgesetzten selbstbezogenen Aktivität ist, muss nicht nur grammatisch, sondern auch empirisch durchgehend und von Anfang an vorhanden sein. Die vom Bundesverfassungsgericht im zweiten Urteil zum Schwangerschaftsabbruch vorgenommene feinsinnige Unterscheidung zwischen »Entwicklung zum Menschen« (bis zur Einnistung) und »Entwicklung als Mensch « (danach) ${ }^{30}$ hebt diese Implikation nicht auf, da auch »entwickelt sich zu« von einem Satzsubjekt prädiziert wird, das schon am Anfangspunkt der Entwicklung existieren muss. Allerdings lässt der Ausdruck »entwickelt sich zu « einen Wechsel des Subjekts zu: Eine Raupe entwickelt sich zu einem Schmetterling. Zur Bezeichnung des Übergangs von einem formlosen Materieaggregat zu einer sortal bestimmten Substanz ist der Ausdruck »sich entwickeln zu « nicht gut geeignet - wohl weil eine bloße Materieansammlung nicht als Subjekt einer formbildenden Aktivität in Frage kommt.

Die beschriebenen Implikationen und Konnotationen hat der Ausdruck "Entwicklung" ganz unabhängig davon, wie sachangemessen sie dem jeweils beschriebenen Vorgang sein mögen.

Bei der Frage, wann ein Embryo zu existieren beginnt, geht es nicht um eine Entwicklungsphase einer Substanz, sondern um deren Anfangspunkt. Unter der Annahme, dass "menschlicher Embryo« das früheste Phasensortal der sortal bestimmten Substanz "Mensch« ist, steht am Anfang dieser Phase eine substanzielle Veränderung. Zur Bestimmung desjenigen Zeitpunkts, zu dem die Substanz in Existenz tritt, trägt die Rede von "Entwicklung« nichts bei. Die grammatischen und semantischen Eigenheiten des Entwicklungs-Idioms helfen nicht dabei, denjenigen Punkt zu fixieren, von dem an die fragliche und sich fortan entwickelnde Entität existiert. Ist aber erst einmal die Angemessenheit des Verbums »sich entwickeln« für die Beschreibung einer substanziellen Ver-

29 »Epigenetisch« heißen zelluläre Prozesse, die zu vererbbaren Veränderungen in der Genexpression führen, aber nicht in der DNA-Sequenz codiert sind. Die Rolle der epigenetischen Prozesse tangiert auch die Angemessenheit des Totipotenzkriteriums: "Insbesondere Erkenntnisse über epigenetische Steuerungsprozesse relativieren dabei die Bedeutung der genomischen Ausstattung. Die Erbsubstanz legt den Möglichkeitsbereich der Entwicklung fest, determiniert aber nicht den späteren Organismus [...]. Dass sich aus einer totipotenten menschlichen Zelle eine Person entwickeln kann, bedeutet somit nicht, dass dieser personale Mensch der Form nach bereits im Keim vorhanden ist.« Huber 2009, 165. Huber stellt hier auf die individuelle Person ab, nicht auf die Entwicklungsfähigkeit zu einem Menschen. Darin kann er sich dadurch bestärkt fühlen, dass auch im ESchG und im StZG von der Entwicklung $\mathrm{zu}$ einem "Individuum« die Rede ist.

30 Vgl. Bundesverfassungsgericht 1975, 252. 
änderung zugestanden, also für die Bezeichnung des Umschlagspunkts (oder der Übergangsphase?) von Nichtsein zu Sein, so sind die Verwirrungen kaum mehr zu vermeiden. Man stelle sich jemanden vor, der die Paradoxie des instantanen In-Existenz-Tretens einer Substanz in einer Welt kontinuierlicher Verläufe bewusst verschleiern will: Er wäre gut beraten gewesen, das Verb »sich entwickeln « zu diesem Zweck zu erfinden, wenn es nicht schon existierte.

Aristoteles selbst hat anders als Tertullian und die römische Glaubenskongregation die Embryonalentwicklung nicht im Sinne des substanzontologischen Spezies- und des Identitätsarguments aufgefasst. Er vertrat vielmehr die Lehre von der Sukzessivbeseelung, derzufolge in der Entwicklung eines menschlichen Embryos die Nährseele, die Tierseele und die vernünftige Seele nacheinander erworben werden. In Thomas von Aquins pointierter Zusammenfassung: »In der Entstehung des Menschen gibt es zuerst ein lebendiges Wesen, dann ein Tier, zuletzt jedoch einen Menschen «. ${ }^{31}$ In der katholischen Theologie war die Lehre der Sukzessivbeseelung lange Zeit vorherrschend, bis sie im 19. Jahrhundert unter Pius IX. aufgegeben wurde.

Aristoteles entschärft die Sukzessivbeseelungsthese durch die Dynamis/Energeia-Lehre, nach der alle Seelenteile zunächst potentiell und dann aktual besessen werden. ${ }^{32}$ Diese Auffassung ändert aber nichts daran, dass Aristoteles zufolge der Mensch nicht schon mit der Befruchtung zu existieren beginnt, denn was nur der Möglichkeit nach vorhanden ist, ist eben noch nicht vorhanden. Sähe man es anders, so würde die argumentative Last, die in der paradoxen Figur "Etwas entwickelt sich zu dem, was es schon ist « vom Potentialitätsargument auf das Identitätsargument verschoben worden war, wieder auf das Potentialitätsargument zurückverschoben. Dieses kann aber die Last allein nicht tragen, denn auch ein Vermögen (potentia) bedarf, anders als eine bloße Möglichkeit (possibilitas), stets eines Trägers. Wenn dieser Träger nicht immer schon existiert hat, muss er aus etwas anderem entstanden sein, denn eine creatio ex nihilo gibt

31 "In generatione hominis prius est vivum, deinde animal, ultimo autem homo.« Thomas von Aquin, Summa Th. 2, 2 q. 64 a. 1. Die Textbasis für die Sukzessivbeseelungsthese bei Aristoteles ist De generatione animalium II 3, 736b: »That then they possess the nutritive soul is plain (and plain is it from the discussions elsewhere about soul why this soul must be acquired first). As they develop they also acquire the sensitive soul in virtue of which an animal is an animal. For e.g. an animal does not become at the same time an animal and a man or a horse or any other particular animal«. Knoepffler argumentiert, dass in der Entwicklung zu einem Menschen nach einer entwicklungsbiologisch informierten aristotelischthomistischen Auffassung sogar vier oder fünf substanzielle Veränderungen vorkommen (vgl. Knoepffler 2007).

$32 »$ For all three kinds of soul, not only the nutritive, must be possessed potentially before they are possessed in actuality.« Aristoteles, GA II 3, 736b. 
es bei Aristoteles nicht. Wenn ein $\tau o ́ \delta \varepsilon \tau \imath$ aus einem nicht- $\tau o ́ \delta \varepsilon \tau \imath$ entsteht, entsteht es nicht aus schlechthin nichts. ${ }^{33}$

Als Zwischenbilanz lässt sich festhalten, dass zur Klärung der Fragen, ob eine totipotente Zelle ein Embryo ist und zu welchem Zeitpunkt ein menschliches Wesen zu existieren beginnt, weder die SKIP-Argumente noch der Rekurs auf die aristotelische Substanzontologie hinreichen.

Nun hat aber die Bestimmung des ontologischen Status totipotenter $\mathrm{Hu}-$ manzellen starke Präjudizwirkung für die ethische Debatte. Insbesondere die Kombination von Spezies- und Identitätsargument ist dazu angetan, eine biound rechtsethische Frage in eine klassifikatorische zu überführen: Wenn eine totipotente menschliche Zelle schon ein Mensch ist, dann ist die Frage ihres normativen Status nicht mehr ernstlich offen: Sie ist dann ein Grundrechtsträger. Der deutsche Gesetzgeber hat sich dieser Statusbestimmung offenkundig nicht angeschlossen, denn wenn Embryonen, wie sie im Embryonenschutz- und im Stammzellgesetz definiert werden, klarerweise unter die Extension von "Mensch" und damit unter die Menschenwürdegarantie des Grundgesetzes fielen, bedürfte es keines auf eine eigene Embryodefinition gestützten Schutzstatus. Da die Art der Beziehungen zwischen totipotenten Humanzellen und menschlichen Individuen aber umstritten ist, waren aufgrund des im Strafrecht geltenden Analogieverbots gesetzliche Regelungen mit stipulativen Legaldefinitionen erforderlich.

\section{Kontinuität und Gradualität: Natura non facit saltus}

Die Lehre, dass Substanzen instantan entstehen, weil sie kein Mehr oder Minder zulassen, hat eine natürliche Antipodin, nämlich die naturphilosophische Kontinuitätsthese. Diese lässt sich in allgemeinster Form so ausdrücken, dass die Natur keine Sprünge macht. Veränderungen in der Natur geschehen der Kon-

33 In De generatione et corruptione I 3 argumentiert Aristoteles, dass eine Substanz sowohl aus einem formlosen Materieaggregat als auch aus etwas entstehen kann, was die fragliche Substanz in geringerem Ausmaß ist. Der zweite Fall, für den die Embryonalentwicklung paradigmatisch ist, lässt sich nicht leicht mit dem »kein Mehr oder Minder «-Prinzip aus der Kategorienschrift vereinbaren, passt aber gut zum schrittweisen Entstehen der Substanz "Mensch" im Sinne der Sukzessivbeseelungslehre (vgl. dazu Carraro 2014, Ch. 3.5.3). In seinen biologischen Schriften zeigt sich Aristoteles an vielen Stellen für Kontinuitätsüberlegungen aufgeschlossen. Eine Möglichkeit, seine These des instantanen Entstehens von Substanzen zu interpretieren, wäre, dass er die Substanz mit dem punktuellen Abschluss einer Phase der kontinuierlichen Eigenschaftsveränderung beginnen lässt. Substanzen sind ja durch ihre jeweils artspezifische Natur ausgezeichnet und diese liegt erst vor, wenn der Erwerb der entsprechenden Eigenschaften abgeschlossen ist. Vgl. am Beispiel der Fertigstellung eines Hauses Pol. I 2, 1252b und Phys. VII 3, 246a. (Auf diese Stellen und diese Interpretationsmöglichkeit hat mich Christof Rapp aufmerksam gemacht.) 
tinuitätsthese zufolge in infinitesimal kleinen Zwischenstufen, die "Stufen« zu nennen strenggenommen schon irreführend ist: Natura non facit saltus. Auch Prozesse, die wir gewöhnlich als diskontinuierliche beschreiben und wahrnehmen, verlaufen bei genauerem Hinsehen nicht sprunghaft. Spätestens das apparateunterstützte Hinsehen belegt, dass es sich nicht am begrenzten Auflösungsvermögen der menschlichen Wahrnehmung bemisst, auf welche Weise Veränderungen in der Natur tatsächlich vonstatten gehen.

$\mathrm{Ob}$ das naturphilosophische Kontinuitätsprinzip ad infinitum gilt, ist bekanntlich in der Physik umstritten; vielleicht macht die Natur im subatomaren Bereich doch Sprünge, was immer das heißen mag. (Dass es kleinstmögliche, also nicht mehr teilbare Dinge gibt, ist zum Beispiel eine andere These als die, dass es kleinstmögliche Veränderungen gibt.) Solange wir uns im Bereich mittlerer Dimensionen bewegen und die Kontinuitätsthese auf die hier zur Debatte stehenden biologischen Prozesse beschränken, können wir diese Fragen auf sich beruhen lassen. Bisher hat niemand behauptet, dass die Hinzufügung eines einzigen Moleküls, Atoms oder subatomaren Teilchens aus einem Nichtembryo einen Embryo macht.

Wie verhält sich die Kontinuitätsthese zur Volksweisheit, dass es ein bisschen schwanger nicht gibt? Als Beispiel für einen Einschnitt, der keine Abstufung zulässt, ist der Beginn einer Schwangerschaft so eindrücklich, dass sich das Diktum auch in anderen Sprachen findet (»You can't be a little pregnant«). Die nächstliegende Harmonisierung von Kontinuitätsprinzip und Nichtabstufbarkeit besteht in der Auffassung, dass nur akzidentelle Veränderungen sich kontinuierlich vollziehen, nicht aber das Entstehen und Vergehen von Substanzen. Die Auffassung, dass Lebewesen instantan entstehen, lässt sich aber mit entwicklungsbiologischen Tatsachen kaum vereinbaren. So ist die Befruchtung einer Ei- durch eine Samenzelle ein Vorgang, der in allen seinen Phasen Zeit konsumiert. Das Eindringen des Spermiums in die Schutzhülle der Eizelle braucht etwa 20 Minuten. Nach dem Durchdringen der Zona pellucida verschmilzt zuerst das Zellplasma beider Zellen (Plasmogamie), bis nach ca. 30 Stunden die Vorkernverschmelzung (Karyogamie) abgeschlossen ist, also die Anordnung der Chromosomen in der Metaphasenplatte. Von dort an spricht man von der "Zygote«. In der weiteren Entwicklung der Zygote zur Morula geht spätestens im 8-Zell-Stadium die Totipotenz verloren. Die Nidation beginnt am 5. oder 6. Tage und gilt Ende der zweiten Woche als abgeschlossen, wenn der Embryo vollständig in die Gebärmutterschleimhaut eingebettet ist. ${ }^{34}$

34 Das Gesetz über den Schwangerschaftsabbruch setzt den Beginn der Schwangerschaft genauer: den Zeitpunkt, von dem an ein Abbruch als Schwangerschaftsabbruch im Sinne dieses Gesetzes gilt - auf den »Abschluß der Einnistung des befruchteten Eies in der Gebärmutter« fest (StGB $\$ 218$ Abs. 1). 
In der philosophischen Rezeption der entwicklungsbiologischen Befunde gibt es keinen Konsens darüber, ob die Kernverschmelzung, die Nidation, die Gastrulation, die Bildung des Neuralrohrs, die Organogenese, das Einsetzen von Gehirnaktivität, die Empfindungsfähigkeit, die extrauterine Lebensfähigkeit, die Geburt oder noch etwas anderes den markantesten und relevantesten Einschnitt darstellen. Die Antworten unterscheiden sich auch darin, wofür genau eine Zäsur gesucht wird: dafür, dass ein Mensch zu existieren beginnt? Dafür, dass die Entwicklung zu einem Menschen begonnen hat? Dafür, dass etwas zu jemand wird? Dafür, dass eine Entität ein menschliches Individuum ist? Dafür, dass sie eine Person ist?

Mir kam es bei meinem knappen Referat der embryologischen Entwicklungskaskade allein darauf an, in Erinnerung zu rufen, dass der vermeintlich instantane Beginn eines Menschenlebens ein komplexer und in allen seinen Teilen zeitkonsumierender Prozess ist. Es bedarf also einer Erklärung dafür, dass der Volksmund jedenfalls den Beginn einer Schwangerschaft als punktuellen Einschnitt behandelt. Naheliegende Erklärungen wären die Unkenntnis der entwicklungsbiologischen Details oder deren praktische Irrelevanz. Wenn die Kenntnisse vorhanden sind, bleibt als Erklärung, dass wir uns in vielen Kontexten für die Details nicht interessieren und in unserer sprachlichen Praxis von den Übergangszonen, zu deren Bezeichnung uns die Worte fehlen, abstrahieren. Das würde bedeuten, dass es allein eine Frage der zeitlichen Feinauflösung bei der Betrachtung ist, ob der Beginn eines menschlichen Lebens ein punktueller Einschnitt ist oder nicht. ${ }^{35}$

Bei der medizinethisch ebenfalls brisanten Bestimmung des Todeszeitpunkts bietet sich ein ähnliches Bild: Biologisch ist das Sterben eines Lebewesens ein Prozess, der im unwiderruflichen Tod endet, aber keinen sekundengenauen Einschnitt kennt, den man natürlicherweise als Kriterium nutzen könnte. Jahrtausendelang haben der letzte Atemzug oder der letzte Herzschlag als Zäsur ausgereicht. Seit wir es mit der schwierigen Frage zu tun haben, ob und wann man einem hirntoten Menschen Organe entnehmen darf, um anderer Menschen Leben zu retten, sehen wir genauer hin. Wir lösen den Prozess des Sterbens zeitlich feiner auf und unterscheiden verschiedene Todesbegriffe. ${ }^{36}$

Hinsichtlich der entwicklungsbiologischen Kontinuitätsthese ist noch auffällig, dass sie mit großem Nachdruck von Autoren vertreten wird, die die $B e$ -

35 »Auflösung« ist ein Begriff aus der Optik, der sich mutatis mutandis auf die »Körnigkeit» der sprachlichen Individuation anwenden lässt: Sprachliche Repräsentationen führen implizit stets einen bestimmten Auflösungsgrad mit sich. Vgl. dazu Keil 2006, bes. 99-104.

36 Es handelt sich nicht allein um eine höhere Auflösung im Auge des Betrachters, denn es kamen Veränderungen auf der Phänomenseite hinzu: Seit der Erfindung der Herz-LungenMaschine lassen sich Sterbensphasen dissoziieren, die vorher in enger zeitlicher Folge auftraten. 
fruchtung als entscheidende Zäsur ansehen. Der Hinweis auf die Stufenlosigkeit der biologischen Entwicklung dient bei diesen Autoren dazu, die Befruchtung kontrastierend von dieser Kontinuität abzusetzen und als singulären Einschnitt auszuzeichnen. Es erhöht freilich die Begründungslasten für Zäsurthesen enorm, wenn die einschlägigen naturphilosophischen (Kontinuitätsprinzip) und erkenntnistheoretischen Argumente (Rolle der Feinkörnigkeit der Individuation) bereits auf dem Tisch liegen und gewürdigt worden sind. ${ }^{37}$ Die Argumentation Ragers ist besonders spannungsvoll, denn er spricht davon, dass auch die Befruchtung sich »als kontinuierliche Abfolge von Ereignissen ${ }^{38}{ }^{8}$ vollziehe, als ganze aber den Beginn des Menschseins markiere, und verteidigt dies mit dem Argument, dass es in der weiteren Entwicklung des Embryos keine Diskontinuität mehr gebe. ${ }^{39}$ Das Kontinuumsargument wird hier selektiv eingesetzt: Was andere Kandidaten für den Beginn des Menschseins disqualifiziert, nämlich die Stufenlosigkeit des entsprechenden Entwicklungsprozesses, der lediglich in der Beschreibung in Einzelereignisse zerlegt werde, soll im Falle der Befruchtung für den Zäsurcharakter irrelevant sein.

Nun geht es in der Debatte um die totipotenzbasierte Embryodefinition nicht um eine Einschätzung des Einschnitts der Befruchtung, sondern darum, den Status einer nicht befruchteten totipotenten Humanzelle zu bestimmen, und dies möglichst mithilfe belastbarer biologischer oder ontologischer Argumente. $\mathrm{Zu}$ dieser Statusbestimmung trägt die Zäsur der Befruchtung nichts bei. Und wenn es neben der Befruchtung ex hypothesi keine weiteren Zäsuren gibt, kann sich die Auszeichnung des Merkmals der Totipotenz jedenfalls nicht auf einen besonderen Einschnitt in einem ansonsten kontinuierlichen Verlauf stützen.

37 Exemplarisch Günther Rager: „Die Aufzählung der Einzelereignisse wird lediglich von unserer Beobachtungsgenauigkeit bestimmt. [...] Es muß [...] klar gesehen werden, daß die beschriebenen Stufen der Kaskade Ergebnis unserer begrifflichen Abgrenzungen, nicht aber der Wirklichkeit selbst sind. Jede 'Stufer folgt kontinuierlich aus den vorausgegangenen Prozessen.« Rager 1994, 77.

38 Ebd.

39 "Jedes Entwicklungsstadium geht kontinuierlich in das folgende über. Es gibt keinen Moment in der Entwicklung, an dem man sagen könnte, hier werde der Embryo erst zum Menschen.« »Es ist immer wieder versucht worden, das Menschsein mit der Reifung des Gehirns beginnen zu lassen. Die Differenzierung des Nervensystems ist aber eines der besten Beispiele dafür, daß sich kein Punkt festlegen lässt, an welchem sprunghaft etwas Neues entsteht.» Rager 1994, 82 und 346. 


\section{Vagheit und Sorites-Schlüsse}

Die sprachliche Seite des Phänomens der kontinuierlichen Verläufe ist die semantische Vagheit, also die Randbereichsunschärfe vieler Ausdrücke der natürlichen Sprache. Vage Ausdrücke ziehen keine scharfe Grenze zwischen den Dingen, auf die sie zutreffen, und denen, auf die sie nicht zutreffen. Die beiden bekannten antiken Beispiele »Haufen« und »kahlköpfig« zeigen, dass vage Ausdrücke Schlüsse von klaren Fällen über unklare Fälle bis hin zur Absurdität erlauben oder zu erlauben scheinen. Im sogenannten Sorites-Paradox wird angenommen, dass beispielsweise 10.000 Sandkörner in der richtigen Anordnung klarerweise einen Haufen bilden. Durch die wiederholte Anwendung der Prämisse »Wenn $n$ Körner ein Haufen sind, dann sind auch $n-1$ Körner ein Haufen « wird darauf geschlossen, dass auch ein einziges Korn ein Haufen sei. Die erste Prämisse des Schlusses ist wahr, wenn $n$ groß genug gewählt wird, die zweite Prämisse klingt sehr plausibel, die Konklusion folgt aus den Prämissen, ist aber abwegig. Also muss etwas schiefgelaufen sein. Es gibt aber bis heute keinen Konsens darüber, worin genau der Fehler besteht. Handelt es sich um einen Fehlschluss? Ist eine der Prämissen falsch? Ist es überhaupt unzulässig, aus Prämissen, die vage Ausdrücke enthalten, zu schließen? In der Sprachphilosophie und in der Linguistik sind eine Reihe von elaborierten Theorien der Vagheit entwickelt worden, die außerhalb dieser Disziplinen kaum bekannt sind. ${ }^{40}$

Semantische Vagheit beruht definitionsgemäß nicht auf empirischer Unkenntnis und kann deshalb nicht durch Ermittlung zusätzlicher Fakten beseitigt werden. ${ }^{41}$ Wir können die Körner einer Sandansammlung zählen und immer noch unsicher sein, ob es sich um einen Haufen handelt. Wir mögen die biologischen Details der Befruchtungs-, der Einnistungs- und der Embryonalphase beliebig genau kennen und immer noch unsicher sein, wann ein Mensch zu existieren beginnt. Das Sandhaufen-Beispiel zeigt, dass das Sorites-Paradox auch in einer »körnigen« Welt bestünde, die keine kleineren Gegenstände als Sandkörner enthielte. Die semantische Vagheit zahlloser Prädikate natürlicher Sprachen, die für das Sorites-Paradox verantwortlich ist, ist unabhängig von der Frage, ob das naturphilosophische Kontinuitätsprinzip auch im Mikrokosmos gilt oder ob wir in einer gequantelten Welt leben.

In der Medizinethik werden Sorites-Schlüsse häufig in Form von Slippery

40 Für einen Überblick vgl. Williamson 1994; Keefe/Smith (Hg.) 1997; Graff/Williamson (Hg.) 2002; Walter (Hg.) 2005; Schöne 2011; Raffman 2013.

41 "To say that an expression is vague (in a broad sense of vague) is presumably, roughly speaking, to say that here are cases (actual or possible) in which one just does not know whether to apply the expression or to withhold it, and one's not knowing is not due to ignorance of the facts." Grice 1989, 177. 
slope-Argumenten diskutiert. Da es auf einem rutschigen Abhang kein Halten gibt, wird die Abhilfe darin gesehen, ihn gar nicht erst zu betreten. Das ist leichter gesagt als getan, denn die erste Prämisse eines Sorites-Schlusses, zum Beispiel » 10.000 Körner bilden einen Haufen« oder "Die freie Verkäuflichkeit von Schmerzmitteln ist keine aktive Sterbehilfe« ist unstrittig wahr und die Vorstellung, man könne irgendeinen ersten Schritt im Räsonnement finden, mit dem die schiefe Ebene (so die übliche Übersetzung von »slippery slope«) betreten wird, gehört selber zum Sorites-Problem, nicht zu dessen Lösung. Auch die Rhetorik des zu verhindernden Dammbruchs, zuweilen zum Dammbruch"Argument" geadelt, trägt nichts dazu bei, den ersten unzulässigen Schritt zu identifizieren. Zum Sorites-Problem gehört, dass es nicht nur zwischen einem Haufen und einem Nicht-Haufen, sondern auch zwischen einem klaren Fall eines Haufens und einem Grenzfall eines Haufens keine scharfe Grenze gibt. Mit anderen Worten: Der Ausdruck "Grenzfall« ist ebenfalls vage. Man nennt dies das Problem der höherstufigen Vagheit. Es ist dieses zweite Abgrenzungsproblem, das im Argument der schiefen Ebene übersehen wird, denn in diesem wird davon ausgegangen, dass es vor der Ebene jeweils ein scharf begrenztes Plateau gibt.

Die Beispiele des Lebensbeginns und -endes zeigen, dass bei der Erhöhung des zeitlichen Auflösungsgrads der Betrachtung vermeintlich scharfe Grenzen sich als unscharf erweisen können. Bei grobkörniger Individuierung des Sterbens erscheint die Grenze zwischen Leben und Tod als scharf; je genauer man hinsieht, desto unschärfer wird sie. Um diesen Effekt zu verstehen, muss man berücksichtigen, wie Erkenntnisfortschritt und semantische Vagheit interagieren. Von wissenschaftlicher Forschung erwartet man gewöhnlich, dass sie zu Präzisionssteigerung führt, nicht zu Präzisionsverlust. Dass sich hier scheinbar der gegenteilige Effekt einstellt, liegt daran, dass man im Anwendungsbereich der Prädikate "schwanger", "Mensch«, »Embryo" und »Tod» im Zuge des Fortschritts der Forschung mehr Details sieht, dabei aber die sprachlichen Ausdrücke, die zur Unterscheidung dieser Details nicht gemacht sind, beibehält. Dadurch treten die Grauzonen und Grenzbereiche, die der fragliche Ausdruck immer schon aufwies, ins Blickfeld. Der Unterschied zum antiken Beispiel besteht darin, dass wir vom Ausdruck "Haufen « immer schon wussten, dass er vage ist, während wir es vom Ausdruck »schwanger « nicht wussten. Ein weiterer Unterschied besteht darin, dass Menschen und Embryonen keine haufenartigen Materieaggregate sind, sondern gegliederte funktionale Ganzheiten. Allgemein sind Sorites-Schlüsse mit Kontinuativa (Massentermini) einfacher zu konstruieren als mit Individuativa (Sortalausdrücken). Da aber die Hinzufügung eines einzelnen Atoms oder Moleküls nicht geeignet ist, aus einem klaren Fall eines 
Nichtembryos einen klaren Fall eines Embryos zu machen, ist der Ausdruck "Embryo" ebenfalls soritisch vage und lässt Grenzfälle zu. ${ }^{42}$

Die Wissenschaft reagiert auf Präzisierungsbedarf oft mit neuer Fachterminologie, das Recht mit stipulativen Legaldefinitionen vorhandener Ausdrücke. In manchen Fällen sind beide Züge nicht angezeigt, weil man die Verbindung zwischen kulturell verankerten begrifflichen und moralischen Intuitionen nicht zu stark lockern möchte. So ist der Ausdruck »Tod « keiner, den man in Abhängigkeit vom aktuellen Regelungszweck einfach stipulativ definieren könnte. Die kulturelle Bedeutung des Todesbegriffs und des mit ihm verbundenen Bündels von Überzeugungen und Einstellungen ist zu stark, als dass man sich legitimerweise über sie hinwegsetzen könnte. Der Gesetzgeber hat mit Bedacht davon Abstand genommen, das Verhältnis zwischen »Tod" und »Hirntod» verbindlich klären zu wollen; der technische Ausdruck »Hirntod « taucht im Transplantationsgesetz überhaupt nicht auf, wiewohl der Sache nach eben davon die Rede ist.

Das durch Vagheit induzierte Sorites-Problem betrifft wohlgemerkt nicht die Frage, wo jeweils die Grenze zu ziehen ist. Vagheit und höherstufige Vagheit haben vielmehr zur Folge, dass, wo auch immer man die Grenze zieht, sich stets Grauzonen und Grenzfälle ergeben. Das Interpolieren von Zwischen- und Vorstufen, ein üblicher Zug beim Umgang mit unscharfen Grenzen, führt deshalb regelmäßig nicht zu einer Beseitigung, sondern zu einer Vervielfachung von Grenzfällen.

Die Exkurse zum naturphilosophischen Kontinuitätsprinzip und zum Sorites-Paradox sollten zeigen, dass die Abgrenzungsprobleme, die sich im ESchG und im StZG stellen, nur ein Spezialfall der allgemeinen Herausforderung des vernünftigen Umgangs mit unaufhebbar unscharfen Grenzen sind. Wie gehen wir im Alltag, in der Wissenschaft, in der Moral und im Recht vernünftigerweise mit Abgrenzungsproblemen um, wenn die Natur der Dinge uns keine scharfen Einschnitte darbietet, an die wir sachangemessene Unterscheidungen knüpfen könnten? Welche Möglichkeiten gibt es, folgenreiche Grenzziehungen in kontinuierlichen Verläufen $\mathrm{zu}$ rechtfertigen ? $^{43}$

42 Nicht alle vagen Ausdrücke sind im gleichen Ausmaß vage. Ferner können Ausdrücke intensional vage sein, ohne extensional vage zu sein - wenn nämlich Grenzfälle noch nicht vorgekommen, aber möglich sind, genauer: wenn der Sinn (Intension) des Ausdrucks nicht von allen möglichen Fällen festlegt, ob sie in seinen Anwendungsbereich (Extension) fallen.

43 Einen Überblick über diesen Problemkomplex verschaffen die im Rahmen des philosophisch-rechtswissenschaftlichen Forschungsprojekts "Vernünftiger Umgang mit unscharfen Grenzen« entstandenen Publikationen. Die Bibliographie ist auf der Seite www.unscharfe-grenzen.de abrufbar. 


\section{Gradualität und Ethik}

Da das Recht der Fortpflanzungsmedizin für eine nichtwillkürliche Beantwortung der Statusfrage auf markante Einschnitte oder Unterschiede angewiesen ist, tut es sich notorisch schwer damit, natürliche Kontinuitäten und semantische Vagheit anzuerkennen. Auch wenn empirisch schwer zu bestreiten ist, dass das Entstehen und das Enden eines menschlichen Lebens Prozesse sind, die sich nicht von selbst in natürliche Einheiten gliedern und für deren Sortierung kaum gemeinsprachliche Sortalausdrücke zur Verfügung stehen, scheinen die rechtlichen und moralischen Kosten dafür, am Lebensanfang und -ende kontinuierliche Übergänge anzunehmen, vielen als untragbar hoch. Die Prominenz des Argumentes der schiefen Ebene in der Bioethik ist ein Indiz für diese Spannung.

Im Strafrecht kommt hinzu, dass auf Vagheit zurückgehende Auslegungsspielräume, die in anderen Rechtsgebieten tolerabel oder sogar zweckmäßig sind, rechtsstaatlich nicht zulässig sind. Embryonenschutzgesetz und Stammzellgesetz gehören zum Nebenstrafrecht und unterliegen damit wie alle Strafgesetze dem Bestimmtheitsgebot: "Eine Tat kann nur bestraft werden, wenn die Strafbarkeit gesetzlich bestimmt war, bevor die Tat begangen wurde $« .{ }^{44} \mathrm{Nach}$ der Rechtsprechung des Bundesverfassungsgerichts erwächst dem Gesetzgeber aus dem Prinzip nulla poena sine lege die Aufgabe, "die Voraussetzungen der Strafbarkeit so konkret zu umschreiben, daß Tragweite und Anwendungsbereich der Straftatbestände zu erkennen sind und sich durch Auslegung ermitteln lassen ${ }^{45}$ Für die strafrechtliche Regelung des Umgangs mit Embryonen und totipotenten Humanzellen bedeutet dies, dass deutlich erkennbare Zäsuren erforderlich sind. Weiterhin gilt im Strafrecht das ebenfalls aus Art. 103, Abs. 2 GG abgeleitete Analogieverbot, das eine für den Täter ungünstige erweiternde (»analoge«) Gesetzesauslegung verbietet. Maßgeblich ist stets, welche Tatbestände durch den Wortlaut des Gesetzestextes erfasst sind. Und schließlich gilt im Recht ein aus dem Gleichbehandlungsgebot (Art. 3, Abs. 1 GG) abgeleitetes Willkürverbot. ${ }^{46}$

Kontinuierliche Übergänge im Phänomenbereich sind aus diesen Gründen für das Strafrecht eine besondere Herausforderung. Dass überhaupt mit so

44 Art. 103, Abs. 2 GG; gleichlautend $\$ 1$ StGB.

45 So legt das Verfassungsgericht den Art. 103 Abs. 2 GG aus (Bundesverfassungsgericht 1989, 374). Das Analogon des Bestimmtheitsgebots im Verfassungsrecht der USA ist die »void for vagueness«-Doktrin, nach der zu vage formulierte Gesetze rechtlich ungültig sind.

46 Das Willkürverbot verbietet nach ständiger Rechtsprechung des Bundesverfassungsgerichts, ohne sachlichen Grund entweder wesentlich Gleiches willkürlich ungleich oder wesentlich Ungleiches willkürlich gleich zu behandeln (vgl. z. B. Bundesverfassungsgericht 1988, 121 und 1960, 246). Es ist auch an die Legislative adressiert, die verfassungswidrig willkürlich handelt, wenn sich für eine gesetzliche Regelung kein sachlicher Grund finden lässt (vgl. Bundesverfassungsgericht 1995, 123). 
großer Energie und so großem Ernst punktuelle Einschnitte gesucht werden, wäre ohne die genannten verfassungsrechtlichen Rahmenbedingungen schwer verständlich. ${ }^{47}$

Was nun das bioethische Kontinuumsargument betrifft, so ist der Hinweis am Platz, dass es nicht ganz glücklich benannt ist. Kontinuität als solche hat dem Argument zufolge gerade keine ethische Relevanz, vielmehr wird die Unmöglichkeit, in einem kontinuierlichen Verlauf einen markanten Einschnitt zu finden, zum Anlass genommen, den stipulierten Anfangspunkt einer kontinuierlichen Entwicklung besonders auszuzeichnen. Das Argument besagt ja, dass der Embryo, weil es in seiner Entwicklung keine moralisch relevanten Einschnitte gebe, schon zu Beginn seiner Entwicklung die moralisch relevante Eigenschaft $\mathrm{X}$ besitzen müsse. Da die Konklusion ohne weitere Prämisse nicht folgt, wird das Kontinuumsargument häufig mit dem tutioristischen Vorsichtsargument kombiniert: Angesichts von Grenz- und Zweifelsfällen sei stets dem sichereren $(t u$ tior) Prinzip zu folgen, auch wenn man dabei die Grenze aus theoretischer Sicht möglicherweise zu weit im Bereich des Erlaubten setzt. Wenn ein so hohes Gut wie die Menschenwürde auf dem Spiel steht, die als normatives Fundament des ESchG und des StZG angeführt wird, ${ }^{48}$ sei ein "error on the safe side» vorzuziehen.

Diese Kombination von Kontinuums- und Vorsichtsargument entspricht recht genau dem, was als Slippery slope-Argument bezeichnet wird; jedenfalls rekonstruiere ich das Slippery slope-Argument im Sinne dieser Kombination. In dialektischen Kontexten, in denen die Frage, wo das sichere Plateau endet, selbst umstritten ist, stoßen Slippery slope-Argumente an ihre Grenzen. Während in der Debatte über die Zulässigkeit des Schwangerschaftsabbruchs als Beginn der abschüssigen Bahn häufig der Zeitpunkt der Befruchtung angesehen wird ${ }^{49}$,

47 Dabei scheint die Restriktion des Willkürverbots für sich genommen die schwächste zu sein. Verfassungsrechtlich wird allgemein argumentiert, dass die Schwierigkeiten, einen markanten Einschnitt in einem Kontinuum zu finden, die Anforderungen an einen »sachlichen Grund " gerade absenken. (Dieser Figur entspricht in der Vagheitsdebatte das Prinzip der semantischen Toleranz: Wenn ein Phänomen ein Grenzfall von F ist, ist es weder sprachwidrig, es als $\mathrm{F}$ zu bezeichnen, noch, es als nicht-F zu bezeichnen.) Demgegenüber steigen die Anforderungen an einen sachlichen Grund wieder durch die Hochrangigkeit des Rechtsguts sowie durch die eklatanten Wertungswidersprüche zwischen ESchG, StZG und dem Gesetz über den Schwangerschaftsabbruch.

48 Ausführlich dazu Kersten 2004, 403-515.

49 Auch bezüglich dieser Grenzziehung wird allerdings bestritten, dass die unbestrittene Kontinuität gemeinsam mit dem Tutiorismus zu ihrer Rechtfertigung ausreicht: »Die fließenden Übergänge, auf die das Kontinuitätsargument hinweist, machen es zweifellos erforderlich, Vorsicht walten zu lassen und menschlichen Lebewesen nicht vorschnell den Würdeschutz abzusprechen. Sie können aber nicht begründen, warum menschliche Embryonen schon vom Zeitpunkt der Kernverschmelzung an als Würdeträger angesehen werden müssen.« Dietrich/Czerner 2013, 497. 
bietet sich in der rechtsethischen Debatte über Totipotenz kein vergleichbar »natürlicher« Einschnitt an.

Da auch nicht befruchtete Zellen totipotent sein können, hat der Gesetzgeber im StZG keine zeitliche Zäsur gesetzt, sondern den Totipotenzstatus als solchen geschützt und seine Legaldefinition von »Embryo « darauf gestützt. Und seit vermutet wird, dass Totipotenz sich auch durch Reprogrammierungstechniken induzieren lässt, ist die neue Streitfrage entstanden, ob temporär totipotente Zellstadien bei der Herstellung von iPS-Zellen unter die Embryodefinition des Stammzellgesetzes fallen oder nicht. Umstritten ist aber auch, ob die durch das StZG geschützte Totipotenz einzelner unbefruchteter Zellen überhaupt noch eng genug mit dem ursprünglichen Schutzgut zusammenhängt, nämlich dem Potential, sich zu einem menschlichen Individuum zu entwickeln.

Slippery slope-Argumente sollen moralische Standards davor schützen, auf einer schiefen Ebene ins Rutschen zu geraten und versuchen deshalb, moralische Unterscheidungen gegen tatsächlich bestehende Kontinuitäten abzuschirmen. Demgegenüber knüpfen Gradualitätsargumente an die Kontinuität als solche moralische Konsequenzen. Man kann zwischen zwei Formen unterscheiden, die man das destruktive und das konstruktive Gradualitätsargument nennen könnte, oder auch das einebnende und das differenzierende.

Im destruktiven, einebnenden Gradualitätsargument wird unter Berufung auf eine bestehende Kontinuität für die Nichtexistenz eines kategorischen moralischen Unterschieds argumentiert. Zwei populäre Beispiele aus der Tierethik und aus der Abtreibungsdebatte: (i) Weil Menschen sich in ihren kognitiven Vermögen von ihren evolutionären Vorläufern oder von ihren nächsten Verwandten nur graduell, nämlich durch eine Reihe minimaler Schritte unterscheiden, gebe es zwischen Menschen und anderen Tieren keine moralisch bedeutsamen Unterschiede. (ii) Weil »die Entwicklung vom Embryo zum Säugling ein gradueller Prozeß ist«, gebe es auch keine »moralisch entscheidende Trennlinie zwischen dem Neugeborenen und dem Fötus «. ${ }^{50}$

Während das Slippery slope-Argument moralische Unterscheidungen gegen natürliche Kontinuitäten immunisiert, parallelisiert das destruktive Gradualitätsargument beide und liefert damit moralische Unterscheidungen außermoralischen Kontinuitätsüberlegungen aus. Von einem aufgeklärten, vernünftigen Umgang mit Kontinuität und Vagheit scheinen beide Argumente weit entfernt. Die destruktive Kraft des einebnenden Gradualitätsarguments beruht auf der ungeprüften Annahme, dass für plausible moralische Unterscheidungen stets scharfe Grenzen zwischen außermoralischen Phänomenen erforderlich seien. Diese Annahme ist allenfalls auf einem Problematisierungsniveau plausibel, auf dem man das Sorites-Paradox nicht im Blick hat. Ein Rückblick auf die Debatte

50 Singer 1994, 186. 
über soritische Vagheit ist hier instruktiv: Da fast alle Ausdrücke der natürlichen Sprache semantisch vage sind, lässt sich allgemein »das Argumentationsschema der Haufen-Paradoxie als Konstruktionsschema benutzen, um begriffliche Unterschiede zwischen beliebigen Dingen einzuebnen «. ${ }^{51}$ Man interpoliert zwischen zwei Phänomenen Alpha und Omega, deren Differenz man einebnen möchte, eine Sorites-Reihe minimal unterschiedlicher Zwischenglieder und schließt, dass es keinen bedeutsamen Unterschied zwischen Alpha und Omega gebe, weil sich in der Sorites-Reihe keine scharfe Grenze ziehen lässt. Diese Argumentationslinie zieht eine falsche Lehre aus dem Sorites-Problem. Soritische Vagheit zeigt mitnichten, dass es keine klaren Fälle von Alpha und Omega gibt. Vielmehr zeigt sie unter anderem, dass die Existenz klarer Fälle mit der Nichtexistenz einer scharfen Grenze vereinbar ist. Dass sich durch die Interpolation von Sorites-Reihen klare Fälle von Alpha als klare Fälle von Omega erweisen $\mathrm{zu}$ lassen scheinen, sollte vernünftigerweise als eine reductio ad $a b$ surdum soritesförmigen Argumentierens gewertet werden. Wenn aber soritisches Argumentieren schon eo ipso unvernünftig ist, dann wird es durch den Einsatz in moralischen Kontexten nicht vernünftiger.

Während destruktive Gradualitätsargumente durch Interpolation von Soritesreihen faktische oder begriffliche Unterschiede einebnen und daraus die Einebnung moralischer Unterschiede ableiten, stellen konstruktive Gradualitätsargumente darauf $\mathrm{ab}$, faktische und moralische Unterschiede parallel $\mathrm{zu}$ gradualisieren. Beispielsweise gibt es verbreitete gradualistische Intuitionen über die Schutzwürdigkeit von Embryonen; viele Menschen finden Spätabtreibungen ethisch weniger vertretbar als frühe. Als Argument werden unter anderem der erreichte Entwicklungsstand des Fötus, insbesondere das Ausmaß seiner Fähigkeiten und der Grad der Unabhängigkeit seiner Weiterentwicklung von der Mutter angeführt. ${ }^{52}$ Konstruktive Gradualitätsargumente folgen einem Je-desto-Prinzip, das in diesem Fall lauten könnte: Je weiter fortgeschritten die Entwicklung, desto größer der Schutzanspruch, welcher gleichsam mitwächst. ${ }^{53}$ Alan Gewirth hat ein entsprechendes Proportionalitätsprinzip formuliert ${ }^{54}$ und dieses auch auf das Mitwachsen einer als abstufbar verstandenen Menschen-

51 Pardey 2006, 28.

52 Birnbacher behauptet allgemein, "dass der common sense von einer gradualistischen Auffassung vom normativen Status ausgeht, während das Potenzialitätsprinzip einen konstanten Status über alle Entwicklungsstadien postuliert«. Birnbacher 2011, 222.

53 Ein solches Prinzip wird auch für das Recht postuliert: „Der Grundrechtsschutz qualifiziert entwicklungsfähiger pränataler Entitäten sollte ferner nach Entwicklungsstufen abgestuft werden. Der anfangs nur schwache Schutz wird mit fortschreitender Entwicklung des Embryos hin zum Menschen im Sinne des Art. 1 Abs. 1 Satz 1 GG fortlaufend intensiviert." Laimböck in diesem Band, 94. Für einen gestuften Schutz des Lebens im Sinne des konstruktiven Gradualitätsarguments plädiert auch Merkel 2002.

54 Gewirth 1978, 121. 
würde angewandt. ${ }^{55}$ An konstruktiven Gradualitätsargumenten ist attraktiv, dass sie eine differenzierende moralische Behandlung von Phänomenen erlauben, die sich in nichtmoralischen Hinsichten nur graduell unterscheiden.

Das Gesetz über den Schwangerschaftsabbruch zieht durch das entwicklungsbiologische Kontinuum eine Grenze von zwölf Wochen, von der an der Abbruch nur noch bei medizinischer Indikation straffrei ist. Als Grund für diese Grenzziehung wird von biologischer Seite angegeben, dass die Entwicklung der Organanlagen und des Zentralnervensystems bis zur zwölften Schwangerschaftswoche im Wesentlichen abgeschlossen seien. Wie belastbar dieser Grund ist, ist umstritten. Das Beispiel zeigt aber ein anderes Problem an: Das Je-destoPrinzip des konstruktiven Gradualitätsarguments ist in vielen Kontexten nicht anwendbar. Seine Anwendbarkeit setzt voraus, dass überhaupt eine dem graduell abgestuften Phänomen korrespondierende Skala von Handlungsoptionen zur Verfügung steht. Sind die Handlungsoptionen gröber abgestuft oder sogar binär (abtreiben oder austragen?), so läuft das gradualistische Je-desto-Prinzip leer. Es läuft auch dann leer, wenn statt der Handlungsoptionen der normative Status, der dem erreichten Entwicklungs- oder Fähigkeitsgrad entsprechen soll, seinerseits aus begrifflichen Gründen nicht gradierbar ist. Letzteres wird zum Beispiel von der Menschenwürde behauptet und verfassungsrechtlich mit Bezug auf deren »Unantastbarkeit» nach Art. 1 GG gerechtfertigt. ${ }^{56}$ Diese Rechtfertigung dürfte indes kurzschlüssig sein. Weder aus der Unantastbarkeit noch aus der Unabwägbarkeit der Würde von Menschen folgt etwas darüber, wie es um den Würdestatus von Wesen bestellt ist, von denen gerade in Frage steht, ob sie (schon) Menschen sind. ${ }^{57}$

Die Spannung zwischen der Je-desto-Logik des konstruktiven Gradualitätsarguments und der Entweder-oder-Logik der Statusbestimmung markiert ein ungelöstes Problem. In unserer Alltagsontologie und im Recht sind »Mensch" und «Embryo « binär kodiert. Ein bisschen Mensch gibt es nicht, mithin muss ein punktueller Beginn gesucht werden. Zum anderen leben wir in einer Welt kontinuierlicher Verläufe, der als Bauplan nicht die sortale aristotelische Substanzontologie zugrunde liegt. Die Idee, dass Entwicklung im Falle von Lebewesen bedeutet, dass etwas zu derjenigen sortal bestimmten Substanz wird, die es ontologisch schon ist, ist eine voraussetzungsreiche und hochproblematische

55 Gewirth 1992, 25.

56 Vgl. dazu Dietrich/Czerner 2013, 509.

57 Pointiert Gutmann: „Das rechtliche Konzept der Menschenwürde, wie es im Grundgesetz und in internationalen Regelungen zu finden ist, ist historisch vielmehr ausschließlich vor dem Hintergrund der Missachtung fundamentaler Rechte geborener Menschen zu verstehen. Demgegenüber kann die These, schon der Wortlaut des Art. 1 Abs. 1 GG (`Würde des Menschen $`$ bedinge, auch embryonale Frühformen des Menschseins unter die Norm zu subsumieren, nicht verfangen." Gutmann 2005, 138. 
philosophische Annahme, auch wenn sie sich in bestimmten Redeformen grammatisch verfestigt hat. Die Verteidigung dieser Annahme kann nicht darin bestehen, ihr entgegenstehende gradualistische Intuitionen zu leugnen.

Auch destruktive Gradualitätsargumente dokumentieren allerdings keinen vernünftigen Umgang mit unscharfen Grenzen. Die unscharf begrenzte Extension eines Ausdrucks durch soritesförmiges Argumentieren immer weiter auszudehnen und damit beliebig große Unterschiede $\mathrm{zu}$ nivellieren ist weder theoretisch befriedigend noch moralisch akzeptabel. Das Sorites-Paradox ist weder Lizenz für theoretischen Defätismus noch für moralischen Nihilismus, sondern Indiz für eine bleibende Herausforderung. Konstruktive Gradualitätsargumente scheinen für diese Herausforderung am besten gerüstet, sind allerdings in manchen Kontexten sachunangemessen oder unanwendbar. Ob die Entstehung eines Embryos zu diesen Kontexten gehört, ist umstritten. Allerdings scheint mir angesichts der großen Schwierigkeiten, das punktuelle InExistenz-Treten von Substanzen überhaupt verständlich und ontologisch plausibel zu machen, der größere Teil der Begründungslast auf Seiten derjenigen zu liegen, die Kontinuitäts- und Gradualitätsprinzipien ablehnen.

\section{Natürlichkeits- und Künstlichkeitsargumente}

Im BMBF-Projekt »Entwicklungsbiologische Totipotenz« sind unterschiedliche Einschätzungen hinsichtlich des Status nicht durch Befruchtung entstandener totipotenter Humanzellen vertreten worden. Unter dem Stichwort »Bestimmung als normatives Kriterium « wird zugunsten forschungsliberaler Positionen insbesondere der hohe Künstlichkeitsgrad von zu Forschungs- und Therapiezwecken technisch manipulierten totipotenten Zellen angeführt. Die Argumentation lautet, dass im Fall der natürlichen Entstehung Totipotenz den Embryonenstatus begründe und eo ipso ethisch relevant sei, während es bei durch Reprogrammierungstechniken induzierter Totipotenz zusätzlicher normativer Kriterien bedürfe, um den Status der Entität zu bestimmen. Es könne »aufgrund des Artifizialitätsgrads bei der Erzeugung totipotenter Zellen die Zwecksetzung für die Statusbestimmung mit-konstituierend sein «. ${ }^{58}$ Dass die Zwecke der medizinischen Forschung und Therapie nicht diejenigen Frankensteins sind, ist nach dieser Auffassung schon für die Statusbestimmung relevant, also für die Definition von »Embryo«. Durch das Kriterium der Natürlichkeit bzw. Artifizialität soll die unerwünschte Konsequenz vermieden werden, dass die Frage, welche Forschung mit den fraglichen Entitäten erlaubt ist, von wechselnden

58 Advena-Regnery et al. 2012, 231. Vgl. auch Advena-Regnery in diesem Band, $223-250$ und zu einem anderen Schluss kommend Dederer et al. in diesem Band, 109-136. 
Zwecken und Forschungsinteressen abhängig gemacht wird: Die Zweckbestimmung sei erst dann für den Status "mit-konstituierend", wenn der hohe Artifizialitätsgrad der Entität den Spielraum dafür eröffnet hat. ${ }^{59}$

Zur Berufung auf die Natürlichkeit oder Künstlichkeit einer Entstehung ist zunächst zu bemerken, dass "Natürlichkeit« verschiedene Antonyme hat. ${ }^{60}$ Die Fortpflanzung ist beim homo sapiens von vornherein kein blinder Naturprozess. Ohne absichtliches menschliches Zutun wird ein solcher Prozess nicht in Gang gesetzt, weder innerhalb noch außerhalb der Petrischale. Versteht man unter einem "natürlichen" Vorgang etwas, was von selbst geschieht und keinerlei menschlichen Zutuns bedarf, dann beginnt die menschliche Fortpflanzung nicht auf natürliche Weise. In einem anderen Sinn von »natürlich" unterscheidet man zwischen natürlicher und künstlicher Befruchtung. In diesem Sinn lässt sich auch zwischen natürlich und artifiziell entstandenen totipotenten Zellen unterscheiden, wobei Advena-Regnery et al. nicht behaupten, dass diese Unterscheidung eine scharfe sei: "Problematisch wäre dabei offensichtlich jedoch eine exakte Grenzziehung zwischen schutzwürdigen snatürlich` entstandenen und schutzlosen rartifiziell entstandenen totipotenten Zellen, gerade mit Hinblick auf das Spektrum an Möglichkeiten der Erzeugung totipotenter Zellen. ${ }^{61}$ Die Unterscheidung zwischen Naturprodukt und Artefakt kann nicht trennscharf sein, weil es unterschiedliche Eingriffstiefen in natürliche Verläufe und entsprechend unterschiedliche "Fertigungstiefen $"$ von Endprodukten gibt.

Es soll nach Advena-Regnery et al. deshalb der hohe Artifizialitätsgrad sein, der bei nicht durch Befruchtung entstandenen totipotenten Zellen das Heranziehen zusätzlicher normativer Kriterien zur Statusbestimmung erlaubt. Hier scheint nun wiederum eine Schwellensetzung erforderlich, gerade angesichts der Vielfalt der möglichen Techniken. Der Künstlichkeitsgrad bei durch Reprogrammierungstechniken induzierter Totipotenz ist zweifellos hoch, während kaum (noch) jemand bestreitet, dass einem durch die IVF-Technik entstandenen Embryo spätestens nach der Einsetzung derselbe Status zukommt wie einem sexuell gezeugten (nach dem ESchG auch schon vorher, weshalb das kryokonservierte Lagern im Vorkernstadium erlaubt ist, das Verwerfen hingegen nicht).

Die Unterscheidung zwischen natürlich entstandener und technisch induzierter Totipotenz scheint einstweilen nicht durch soritische Vagheit herausgefordert. Einschlägiger ist ein Phänomen, das man in der Vagheitsdebatte die »kombinatorische Vagheit» von Bündelbegriffen genannt hat. ${ }^{62}$ Bündelbegriffe

59 Allgemein gelte nämlich: »Der Status einer Entität sollte sich so weit wie möglich anhand ihr eignender beschreibbarer Qualitäten ableiten lassen, um nicht der Willkür von Handlungen und Absichten Dritter ausgesetzt zu sein.« Advena-Regnery et al. 2012, 231.

60 Vgl. z. B. Birnbacher 2006, 1- 16.

61 Advena-Regnery et al. 2012, 231.

62 Vgl. Alston 1967, 219. 
sind solche, die nicht über eine Menge notwendiger und gemeinsam hinreichender Bedingungen definiert sind, sondern über die Erfüllung von Merkmalen oder Merkmalskombinationen von einer heterogenen und vielleicht offenen Liste. Möglicherweise ist "Embryo" mittlerweile ein solcher Bündelbegriff. Gemäß den Legaldefinitionen des ESchG und des StZG genügt für den Embryonenstatus totipotenter Zellen eine funktionale Äquivalenz mit unstrittigen Embryonen, nämlich eine des Entwicklungspotentials, während eine ätiologische Äquivalenz nicht erforderlich sei. Es gäbe mithin mindestens zwei voneinander unabhängige Kriterien - durch Befruchtung entstanden und totipotent -, von denen umstritten ist, ob beide für den Embryonenstatus notwendig sind oder nur eines. Zusätzlich kompliziert wird die kriteriale Lage dadurch, dass für den Fall reprogrammierungsinduzierter Totipotenz das ätiologische Kriterium im Vorschlag der BMBF-Forschungsgruppe nicht einfach gestrichen, sondern durch ein anderes ersetzt wird, nämlich den Erzeugungszweck. In nuce: Totipotenz reprogrammierter Somazellen plus Klonierungszweck konstituiert Embryo, Totipotenz plus Forschungszweck nicht.

Die Auffassung, dass im Fall der natürlichen Entstehung Totipotenz den Embryonenstatus begründet, während es im artifiziellen Fall zur Statusbestimmung des zusätzlichen normativen Kriteriums der Zwecksetzung bei der Erzeugung bedarf, erscheint mir argumentativ etwas instabil. Im folgenden Abschnitt versuche ich zu zeigen, dass das angeblich zusätzliche, ergänzende Kriterium gravierendere Rückwirkungen auf die kriteriale Rolle der Totipotenz hat als seine Proponenten annehmen.

\section{Was bleibt von der normativen Relevanz der Totipotenz?}

Wenn man dem Gesetzgeber, der an Reprogrammierungstechniken noch nicht dachte, darin folgt, dass totipotente Zellen Embryonen und damit menschliche Wesen sind, also Grundrechtsträger, so werden sie durch den ersten Artikel unserer Verfassung geschützt. Eine Verletzung des unabwägbaren Rechtsgutes der Menschenwürde kann nicht dadurch gerechtfertigt werden, dass im Zuge dieser Verletzung gleichzeitig geringerwertige Güter wie Forschungsfortschritt oder neue Therapiemöglichkeiten erreicht werden. Wenn also die Absicht oder begründete Aussicht, diese geringeren Güter zu erreichen, ausreicht, um die Erzeugung von und die Forschung an totipotenten Zellen zu rechtfertigen, dann kann der Wert dessen, was da verletzt wird, so groß nicht gewesen sein. Dann kann die Totipotenz einzelner Zellen, ob der Gesetzgeber sie nun »Embryonen« nennt oder nicht, rechtsethisch nicht so relevant sein wie es nach gegenwärtiger Gesetzeslage der Fall ist, und zwar weder im natürlichen noch im artifiziellen Fall. 
Es handelt sich bei »Natürlichkeit der Entstehung« und »Erzeugungszweck» bei Lichte besehen nicht um »weitere, ergänzende Kriterien [...] neben dem Merkmal der Totipotenz « ${ }^{63}$, sondern um solche, die das Totipotenzkriterium `trumpfen`. Der Vorschlag ist mithin keine konservative Erweiterung bestehender Kriterien für den Embryonenstatus, sondern nötigt dazu, das Verhältnis der Merkmale Totipotenz, Natürlichkeit, Zwecksetzung und wohl auch Entwicklungsstadium von Grund auf neu auszutarieren. Dies erscheint auch in der Sache geboten. Die Relevanz des Totipotenzkriteriums wird offenkundig in der geltenden Rechtslage systematisch überschätzt. Dafür sprechen nicht zuletzt die diskutierten Kontinuitäts- und Vagheitsprobleme, die ein Einfallstor für willkürliche Festsetzungen bieten. ${ }^{64}$

Die Auffassung, dass bereits pränidative Entitäten Träger von Grundrechten sind, macht es im Übrigen schwer erklärlich, warum die hohe natürliche Abortrate zwischen Befruchtung und Einnistung auch von den Vertretern eines uneingeschränkten Lebensschutzes nicht als ethisches Problem angesehen wird. Es wird geschätzt, dass bis zu $50 \%$ der befruchteten Eizellen in frühen Phasen der Schwangerschaft mit subklinischem Verlauf, also unbemerkt, zugrunde gehen. In den meisten Fällen geschieht dies wegen chromosomalen Aberrationen; in diesen Fällen ist die Entwicklungsfähigkeit nicht gegeben. Aber neben diesen fetoplazentaren Gründen können maternale Gründe und äußere physische Einwirkungen für den Spontanabort verantwortlich sein. In diesen Fällen könnte man die betreffenden Menschenleben im Prinzip retten, indem man die gefährdeten Zygoten explantiert und von einer anderen Frau austragen lässt.

Das Szenario erscheint bizarr, aber weshalb? Die Kommentatoren des ESchG halten den Hinweis auf »den verschwenderischen Umgang, den die Natur mit beginnendem menschlichen Leben pflege«, für wenig gewichtig, da "sich die Natur - anders als der Mensch - nicht zu verantworten braucht «. ${ }^{65}$ Mein Szenario, das den Menschen in Garantenstellung versetzt, hat die Funktion einer moralischen Intuitionspumpe. Es ließe sich eine Gesellschaft denken, die es für eine moralische oder rechtliche Pflicht hält, Frauen im fertilen Alter einem zeitlich engmaschigen Massenscreening $\mathrm{zu}$ unterwerfen, auf bestehende Schwangerschaften zu untersuchen, gefährdete befruchtete Eizellen oder Embryonen zu explantieren und ihnen, sofern reproduktionstechnisch möglich, in vivo oder in vitro zur Entwicklung zu verhelfen. Dass einem solchen Szenario zahlreiche außermoralische Gründe entgegenstehen, steht außer Frage. Wenn

63 Advena-Regnery et al. 2012, 229.

64 So bilanziert Jens Reich, »dass das empirische Kriterium der Totipotenz für die Angehörigkeit der metaphysischen Zugehörigkeit eines Objekts zur Menschheit nicht geeignet ist, weil es bei der Beurteilung des frühen vorgeburtlichen Lebens willkürlich gestaltbar und im Streitfall weder nachweisbar noch klar widerlegbar ist.« Reich 2004, 115.

65 Günther/Kaiser/Taupitz 2008, Einf. B, Rn. 42. 
eine entsprechende Pflicht auch in moralischer und rechtsethischer Hinsicht als absurd erscheint, ${ }^{66}$ wäre dies ein Indiz dafür, dass nicht nur die Totipotenz einzelner Zellen, sondern nicht einmal die Existenz durch Befruchtung entstandener entwicklungsfähiger Zygoten schon eo ipso diejenigen Schutzansprüche und Hilfspflichten begründet, die hinsichtlich jedes geborenen Kindes selbstverständlich sind, mit etwas geringerem gesellschaftlichen Konsens auch hinsichtlich von Föten in weit fortgeschrittenen Schwangerschaften. Diese Asymmetrie der moralischen Intuitionen scheint durchaus etwas mit dem erreichten Entwicklungsstand $\mathrm{zu}$ tun $\mathrm{zu}$ haben. Man denke daran, dass bei hochschwangeren Unfallopfern durchaus manchmal versucht wird, durch Explantation das Leben des ungeborenen Kindes zu retten. ${ }^{67}$

Es gibt also verbreitete und nicht leicht von der Hand zu weisende gradualistische Intuitionen über Schutzwürdigkeit, die das Totipotenzkriterium nicht erklären kann. Auch von der deutschen Rechtsordnung wird Totipotenz nicht so konsequent geschützt, wie es der abwägungsfeste Menschenwürdeschutz des Grundgesetzes, der aufgrund der totipotenzbasierten Embryodefinition und der postulierten Statusgleichheit von Embryo und geborenem Menschen unmittelbar greifen müsste, erfordert. Den Embryo in utero schützt die Rechtsordnung nur noch auf dem Papier ${ }^{68}$ und in vitro dürfen seine Zellen seit der Änderung des ESchG 2011 in bestimmten Fällen entnommen und einer zerstörenden Untersuchung im Rahmen einer PID unterzogen werden.

\section{Fazit und Ausblick}

Der Gesetzgeber hat es für zweckmäßig gehalten, im ESchG (\$ 8.1) und im StZG (\$ 3.1) zwei sowohl vom allgemeinen als auch vom entwicklungsbiologischen Sprachgebrauch abweichende Rechtsbegriffe des Embryos zu definieren (»Als

66 Ein Grund dafür ist sicherlich die moralische Asymmetrie zwischen positiven Hilfspflichten und Schädigungsverboten: Erstere gelten als »unvollkommene« Pflichten im Sinne von Kant, letztere als "vollkommene«. Aus einem Verbot, Zygoten oder Embryonen zu schädigen oder zu töten, folgt nicht, dass auch eine Pflicht dazu besteht, sie mit beliebig hohem Aufwand zu erhalten.

67 Dass für die moralische Beurteilung der erreichte Entwicklungsstand und die Lebenschancen des Fötus eine große Rolle spielen, zeigt der seinerzeit hochkontroverse (und letztlich erfolglose) Versuch, das »Erlanger Baby« zu retten, einen erst vier Monate alten Fötus, dessen Mutter nach einem Autounfall hirntot war. Strafrechtlich wurde übrigens seinerzeit diskutiert, ob die behandelnden Ärzte, die durch den Anschluss der hirntoten Frau an eine HerzLungen-Maschine in Garantenstellung geraten waren, sich durch ein Beenden der Behandlung eines Schwangerschaftsabbruchs durch Unterlassen schuldig gemacht hätten.

68 »Wenn das geltende Fristen- und Beratungsmodell verfassungsmäßig ist, kann der Embryo nicht Träger des Grundrechts aus Art. 1 Abs. 1 GG sein.« Gutmann 2005, 143; ähnlich Merkel $2002,52 \mathrm{ff}$. 
Embryo im Sinne dieses Gesetzes gilt...«). Indem er Totipotenz zum definierenden Merkmal von »Embryo" erhob, hat er die eingeführte embryologische Terminologie mitsamt ihrer differenzierenden Merkmale souverän ignoriert, also die Phasendifferenzierung nach Zygote, Morula, Blastozyste, Embryoblast, Embryo und Fötus.

Darf der Gesetzgeber das? Freilich darf er das. Legaldefinitionen sind ein übliches, unverzichtbares und legitimes Instrument der Gesetzgebung. In diesem Fall führen die Legaldefinitionen allerdings dazu, dass die Antwort auf die substanzielle rechtsethische Streitfrage, was man mit den fraglichen Gebilden tun darf und was nicht, de facto präjudiziert wird. Wenn totipotente Zellen Embryonen und Embryonen menschliche Wesen sind, schreibt sich, überspitzt formuliert, der Rest des Gesetzes von selbst. Ob die totipotenzbasierten Embryodefinitionen sich auf entwicklungsbiologische, philosophische oder andere Argumente zur Statusfrage stützen können, die so belastbar wären, dass sie das strafbewehrte Verbot derjenigen Forschung an totipotenten Zellen rechtfertigen, die auf Therapie schwerer Krankheiten abzielt, ist äußerst zweifelhaft. Mit »belastbar" meine ich solche Argumente, die nicht von hochumstrittenen weltanschaulichen oder religiösen Annahmen abhängen. Jenseits solcher Annahmen stützen sich die beiden Embryodefinitionen auf selektiv rezipierte Entwicklungsbiologie sowie implizit auf Prinzipien der aristotelischen Substanzontologie, die auch den SKIP-Argumenten zugrunde liegen.

Die paradoxe Figur "Etwas entwickelt sich zu dem, was es schon ist" kann nicht als ein belastbares Fundament gelten. Auch die Auffassungen, dass alle Substanzen sortal bestimmt sind und dass substanzielle Veränderungen - also das Entstehen und Vergehen von Lebewesen - sich nicht kontinuierlich vollziehen, tragen zur Bestimmung des Zeitpunkts, von dem an ein Mensch existiert, nichts bei. Allerdings implizieren sie, dass es einen solchen durch die Natur der Dinge, nämlich durch die artspezifische Natur des Menschen bestimmten Zeitpunkt geben muss, und liegen damit im Konflikt mit der naturphilosophischen Kontinuitätsthese und mit - destruktiven wie konstruktiven - Gradualitätsargumenten.

Dass das ESchG und das StZG eine sortale Ontologie voraussetzen, ist für sich genommen kein besonders starker Einwand, denn nicht nur die aristotelische, sondern auch unsere Alltagsontologie ist eine Ontologie der sortal bestimmten Einzeldinge und ihrer wesentlichen, also artzugehörigkeitsdefinierenden Eigenschaften. Diese Ontologie hat sich in die Grammatik der meisten natürlichen Sprachen so tief eingegraben, dass man ihre Systemzwänge leicht mit solchen des Seienden selbst verwechseln kann. ${ }^{69}$ Von diesen Systemzwängen freie

$69 »[\mathrm{O}]$ rdinary men live so completely within the house of the Stagyrite that whatever they see out of the windows appears to them incomprehensible and metaphysical." Peirce 1931, vii. 
nichtsortale Ontologien stehen außerhalb der akademischen analytischen Metaphysik nicht zur Verfügung. ${ }^{70}$ Auch Moral und Recht werden in der Begrifflichkeit einer sortalen Ontologie betrieben.

»Embryo" gilt als Phasensortal wie »Jugendlicher« oder »Greis«. Die Sortierungsleistung solcher Ausdrücke ist zweigeteilt. Das (kontextuell mitverstandene) Merkmal »menschlich « drückt die Spezieszugehörigkeit aus, "Embryo» grenzt die zeitliche Phase der bereits sortal bestimmten Substanz ein. Der Ausdruck »Embryo« erbringt mithin nicht selbst die moralisch relevante Sortierungsleistung, sondern »erbt« sie von derjenigen Substanz, als deren Phase ein Embryo gilt. Diese Erbschaft des Phasensortals, die auch im Speziesargument ausgebeutet wird, ist so weit unkontrovers, wie von unkontroversen Embryonen die Rede ist. Pränidative totipotente Zellen und Zellverbände sind aber keine unkontroversen Embryonen. Ihr Status ist allerdings nicht deshalb umstritten, weil die Spezieszugehörigkeit der Vollform »Mensch« unklar wäre, sondern weil unklar ist, wann genau ein Mensch zu existieren beginnt - und entsprechend unklar, welche Entität die erste Phase eines Menschen ist. ${ }^{71}$

Wird nun ein totipotenzbasierter Rechtsbegriff des Embryos ohne belastbare biologische oder ontologische Argumente stipulativ definiert, so hat dies aus moralphilosophischer Sicht einen Preis: Die Definition kann nicht mehr an moralische Intuitionen über Schutzwürdigkeit anknüpfen, die mit einem vortheoretischen Alltagsverständnis von "Embryo" verbunden sind. Diese Anknüpfung scheint dem Gesetzgeber nicht ganz unwichtig gewesen zu sein. Er hätte auf den Ausdruck »Embryo« verzichten und das ESchG »Gesetz über den Schutz befruchteter Eizellen« nennen können und das StZG »Gesetz über den Schutz totipotenter Humanzellen«. Dass er dies nicht getan hat und in den Namen beider Gesetze die Bezeichnungen »Embryo" und »embryonal« unterbringt, dürfte nicht nur stilistische, sondern auch rechtspolitische Gründe haben. Zellen und Zellverbände, für die Biologen andere Bezeichnungen haben, "Embryonen « zu nennen hat plausiblerweise einen für die Gesetze akzeptanzerhöhenden Effekt. Das lebensweltliche Verständnis von »Embryo« ist anhand wesentlich weiter entwickelter Embryonen gewonnen worden - anhand solcher, die etwa eine »Embryonalhaltung « einnehmen können, was bei Ein-, Zwei- oder Vierzellern nicht möglich ist. Die moralischen Intuitionen über die Schutz-

70 Für den Unterschied zwischen sortalen und nichtsortalen Ontologien und die Implikationen dieses Unterschieds für die Vagheitsdebatte vgl. Keil 2013, 160 - 162.

71 Der von Vertretern eines uneingeschränkten Lebensschutzes häufig verwendete Ausdruck "menschliches Leben« anstelle des Substanzsortals »Mensch» ist geeignet, diesen Zusammenhang unkenntlich werden zu lassen. »Leben « ist anders als »Mensch « keine aristotelische Substanz und die Bejahung der zweigeteilten Frage, ob eine Entität (a) menschlich und (b) lebendig ist, erweist nicht, dass die Entität ein Mensch ist. Auch ein schlagendes Herz ist menschlich und lebendig. 
würdigkeit von Embryonen dürften sich aus diesem Alltagsverständnis "paradigmatischer" Embryonen speisen. ${ }^{72}$

Die Gleichsetzung von Totipotenz mit »Entwicklungsfähigkeit zu einem Individuum « im ESchG und im StZG lässt leicht vergessen, dass der zellbiologische terminus technicus "Totipotenz « nur einen kontroversen Spezialfall von Entwicklungsfähigkeit bezeichnet. Aus etlichen im technischen Sinn totipotenten Zellen kann kein Mensch werden ${ }^{73}$ während in der natürlichen Embryogenese die Zelleigenschaft der Totipotenz im Achtzellstadium wieder verlorengeht. Weiter entwickelte Embryonen, denen als Ganzen das Potential zukommt, ein voll entwickelter Mensch zu werden, bestehen nicht aus totipotenten Zellen. Die moralischen Intuitionen hinsichtlich der Schutzwürdigkeit hängen aber plausiblerweise an der Entwicklungsfähigkeit zu einem Menschen, nicht an zellbiologischen Merkmalen derjenigen Entitäten, die das ESchG und das StZG schützt.

Mit nur wenig Übertreibung kann man sagen, dass ein auf das Zellmerkmal der Totipotenz gestütztes Potentialitätsargument seine eigene reductio ad absurdum in sich trägt, denn durch die Vielfalt der biomedizinischen Techniken der Totipotenzinduktion whaben sich die Koordinaten, innerhalb deren der herkömmliche Begriff der Potenzialität und das Potenzialitätspinzip sinnvoll anwendbar ist, grundlegend verschoben ${ }^{74}$, so dass die am biologischen Normalfall gewonnenen Intuitionen zur Statusfrage keinen Halt mehr finden. Diese Veränderung der Geschäftsgrundlage unserer Intuitionen betrifft nicht allein das Potentialitätsargument, sondern auch die Individuations- und Persistenzbedingungen menschlicher Lebewesen. ${ }^{75}$

Angesichts der verhärteten Fronten im seit langem andauernden Streit über den Status früher Embryonen und pränidativer Entitäten erscheint die Erwartung nicht realistisch, dass entwicklungsbiologische Befunde oder ontologische Argumente die Statusfrage dereinst konsensfähig entscheiden werden. Dies liegt nicht daran, dass zu wenige Kriterien zur Verfügung stünden, sondern daran,

72 Hier weicht der allgemeine Sprachgebrauch vom entwicklungsbiologischen ab. Was eine Embryonalhaltung einnehmen kann, ist biologisch bereits ein Fötus.

73 S. o., Fn. 11.

74 Birnbacher 2011, 226. Ähnlich argumentiert Hucklenbroich: Durch die Erkenntnis, dass sich gewöhnliche Somazellen in einen totipotenten Zustand zurückprogrammieren lassen, ist »die einzigartige Stellung bezüglich des Entwicklungspotentials, die in unserem intuitiven Paradigma den embryonalen Zellen zugesprochen wird, aufgehoben«. Hucklenbroich 2003, 53.

$75 »$ Das herkömmliche intuitive Paradigma beruht auf bestimmten Auffassungen über die Bedingungen der Individuation, über die Kontinuität der ontogenetischen Entwicklung und über das Entwicklungspotential embryonaler und nichtembryonaler Zellen [...], [die] schon aufgrund der bislang bekannten biologischen Fakten [...] nicht mehr aufrechterhalten werden können.« Hucklenbroich 2003, 37. 
dass ihr kriteriales Gewicht unterschiedlich eingeschätzt wird. ${ }^{76}{ }^{\text {}}$ Embryo« ist nicht nur ein »essentially contested concept « im Sinne von Gallie, sondern erfüllt auch das für semantische Vagheit definierende Merkmal, dass die Anwendungsunsicherheit nicht durch Kenntnis zusätzlicher Fakten beseitigt werden kann. $^{77}$

Dass es kein biologisches oder ontologisches fact of the matter geben soll, unter genau welchen Bedingungen eine Entität die erste Phase eines Menschen ist, mag ein verstörender Befund sein. Wenn aber die semantische Vagheit unserer Substanzsortale kein bloß epistemisches Defizit widerspiegelt, sondern eine Unbestimmtheit in rebus, ist diese Konsequenz nicht zu vermeiden. ${ }^{78}$

Aus moralphilosophischer Sicht spricht der Umstand, dass keine verbindliche Klärung des ontologischen Status pränidativer Entitäten, insbesondere der technisch erzeugten, erreichbar scheint, nicht dagegen, den Streit über ihren moralischen Status mit Argumenten fortzusetzen. Philosophen sind es gewohnt, sich durch jahrhundertelang ausbleibenden Klärungsfortschritt nicht entmutigen zu lassen. In der Literatur mehren sich aber seit geraumer Zeit die Stimmen, die einen dezisionistischen Umgang mit der Statusfrage für unvermeidlich halten oder sogar empfehlen (»Entscheidung « $^{79}$, "soziale Übereinkunft $~^{80}$, "letztlich willkürliche normative Setzung “ ${ }^{81}$ ). Diese Stimmen veranschlagen die argumentativen Ressourcen für die Klärung normativer Fragen geringer als die meisten Fachphilosophen. Die normative Ethik verfügt über einige Übung und auch über ein gewisses Instrumentarium dafür, konfligierende Auffassungen mithilfe von Meta-Prinzipien in ein Überlegungsgleichgewicht zu bringen. Es ist nicht ausgemacht, dass für eine produktive moralphilosophische und rechtsethische Diskussion des Umgangs mit pränidativen Entitäten eine definitive Klärung der ontologischen Statusfrage erforderlich ist. In den Debatten über die Statusfrage wird ohnehin, wie in der Literatur moniert worden ist, "nicht selten versucht, durch deskriptive Ausgrenzung oder Einbeziehung von vornherein normative Weichen $\mathrm{zu}$ stellen . $^{82}$

Eine Lehre aus dieser Debattenlage könnte sein, dass der Streit dorthin zu

76 »Die zugezogenen Kriterien bleiben strittig, weil die zugrunde liegenden embryologischen Fakten unterschiedlich gewichtet werden." Reich 2004, 118.

77 S. o., Fn. 41.

78 Zur Debatte über »ontologische« oder »metaphysische« Vagheit vgl. Keil 2013.

79 »Es lässt sich schlussfolgern, dass keine noch so präzise begriffliche Darstellung die grundlegende Entscheidung überflüssig machen kann, wann der sich entwickelnde `Keim` ein >Mensch ist." Reich 2004, 118.

$80 »$ Konstatiert man die prinzipielle Unlösbarkeit der Statusfrage, so kann ein normatives Urteil über totipotente menschliche Entitäten immer nur Ergebnis sozialer Übereinkunft sein [...]«. Huber 2009, 167.

81 Wiesemann 2003, 149.

82 Günther/Kaiser/Taupitz 2008, § 8 Rn. 2. 
tragen und dort auszutragen ist, wo seine Quellen liegen: Erforderlich sind genuin moralische und rechtsethische Argumente, die offen als solche ausgewiesen werden. Ralf Stoecker hat in der Hirntoddebatte dafür argumentiert, dass wir keine verbesserte Todesdefinition brauchen, sondern eine differenzierte Ethik für den Umgang mit Sterbenden, die argumentativ auf eigenen Füßen stehen muss. ${ }^{83}$ Diese Auffassung verbindet Stoecker mit der Diagnose, dass es sich beim Begriff des Todes um einen Bündelbegriff handelt (also um einen kombinatorisch vagen) und dass mit einzelnen Fasern dieses Bündels unterschiedliche ethische Implikationen und Bewertungen verbunden sind ${ }^{84}$

Analog ließe sich argumentieren, dass wir keine bessere Embryodefinition brauchen, sondern eine differenzierte Ethik des Umgangs mit entstehenden Menschen und mit totipotenten Humanzellen. Viel spricht dafür, dass auch der Begriff des Embryos (mittlerweile) ein Bündelbegriff ist, der zumindest die beiden voneinander unabhängigen Kriterien "durch Befruchtung entstanden" und »entwicklungsfähig zu einem Individuum « umfasst. Wird nun das auf unstrittige Embryonen angewandte Merkmal »entwicklungsfähig zu einem Individuum « zum Merkmal »totipotent « verengt und auf strittige Kandidaten (von prä-embryonalen Zygoten bis hin zu reprogrammierten Somazellen) angewandt, so führt diese Verengung der Intension zu einer Ausweitung der Extension des Embryobegriffs, für die dem Gesetzgeber, um es zu wiederholen, belastbare Argumente fehlen. Eine Ausarbeitung der Idee, dass man unterschiedliche normative Implikationen vernünftigerweise mit einzelnen Fasern des Bündelbegriffs des Embryos verknüpft, muss einer anderen Gelegenheit vorbehalten bleiben.

Für die Fortführung der moralphilosophischen und rechtsethischen Debatte jenseits der Statusfrage scheint es insbesondere erforderlich, das Verhältnis zwischen zwei konkurrierenden Metaprinzipien näher zu untersuchen, nämlich des Tutiorismus und des Liberalismus. Von Ersterem war bereits die Rede, von Letzterem nicht. Dem Vorsichtsprinzip zufolge ist es zum Schutz derart hochwertiger Rechtsgüter wie der Menschenwürde und des Lebensrechts geboten, in Zweifelsfällen einen angemessenen Sicherheitsabstand zum moralisch klarerweise Unzulässigen einzuhalten. Das Liberalitätsprinzip besagt, dass in freiheitlichen Gemeinwesen nicht die Gewährung von Freiheiten begründungsbedürftig ist, sondern der Eingriff in sie, zumal der gesetzlich mit Strafandrohung

83 »Wofür ich plädiere ist also, die Frage, ob und unter welchen Umständen man Menschen Organe entnehmen darf, als eine ethische Frage ernst zu nehmen, ohne darauf bauen zu können, sie dadurch beantworten zu können, dass man klärt, wann ein Mensch tot ist.« "[Wir müssen] uns ganz davon verabschieden, dass uns der vertraute dicke Begriff des Todes für die Frage, wie wir mit Hirntoten umgehen sollen, weiterhelfen kann.« Stoecker 2010, L und XLIX.

84 Stoecker 2010, XXXVI. 
versehene. Dabei steht außer Frage, dass die Wissenschaftsfreiheit, die Berufsund Therapiefreiheit sowie die Reproduktionsfreiheit ${ }^{85}$ dort ihre Grenze finden, wo andere in ihrer Menschenwürde oder ihrem Lebensrecht verletzt werden. $\mathrm{Ob}$ diese Rechtsgüter gefährdet sind und $o b$ es sich bei totipotenten Zellen um Grundrechtsträger handelt, entscheidet aber nicht der Verfechter des Vorsichtsprinzips. Der Tutiorismus hat nicht selbst Verfassungsrang, erst recht ist er kein Freibrief für soritesförmiges Argumentieren. Ferner kann über die Größe des einzuhaltenden Sicherheitsabstands mit Gründen gestritten werden, wobei diese Größe gegen andere Güter wie die begründete Aussicht auf Therapie schwerer Krankheiten abgewogen werden muss.

\section{Literaturverzeichnis}

Advena-Regnery, B./Laimböck, L./Rottländer, K./Sgodda, S.: Totipotenz im Spannungsfeld von Biologie, Ethik und Recht. In: Zeitschrift für medizinische Ethik, (3) 2012, $217-236$.

Alston, W. P.: Vagueness. In: Edwards, P. (Hg.): Encyclopedia of Philosophy. New York 1967, Vol. 8, $218-221$.

Aristoteles: De generatione animalium / On the Generation of Animals. Transl. by A. Platt, New York 1910.

Aristoteles: De generatione et corruptione / Über Werden und Vergehen. Übers. von T. Buchheim, Hamburg 2011.

Aristoteles: De Interpretatione / Lehre vom Satz. Übers. von E. Rolfes, Hamburg 1995. Aristoteles: Kategorien. Übers. von E. Rolfes, Hamburg 1995.

Aristoteles: Physik. Übers. von H. G. Zekl, Hamburg 1995.

Aristoteles: Politik. Übers. von E. Rolfes, Hamburg 1995.

Birnbacher, D.: Natürlichkeit. Berlin/New York 2006.

Birnbacher, D.: Das Potenzialitätsprinzip: Probleme und Paradoxe. In: Lumer, C./Meyer, U. (Hg.): Geist und Moral. Analytische Reflexionen für Wolfgang Lenzen. Paderborn 2011, 223-240.

Bundesverfassungsgericht: Beschluß vom 15. Dezember 1959- 1 BvL 10/55. In: Mitglieder des Bundesverfassungsgerichts (Hg.): Entscheidungen des Bundesverfassungsgerichts. Bd. 10. Tübingen 1960, 234-250.

Bundesverfassungsgericht: Urteil vom 25. Februar $1975-1$ BvF 1, 2, 3, 4, 5, 6/74. In: Mitglieder des Bundesverfassungsgerichts (Hg.): Entscheidungen des Bundesverfassungsgerichts. Bd. 39. Tübingen 1975, 1 - 95.

Bundesverfassungsgericht: Beschluß vom 26. April 1988-1 BvL 84/86. In: Mitglieder des

85 Das "Recht auf reproduktive Autonomie« (Dworkin) ist aus liberaler Sicht negativ als Schutz vor staatlicher Bevormundung auszulegen: „Es geht allein darum, nicht durch ein gesetzliches Verbot an der Wahrnehmung des Selbstbestimmungsrechts in Fragen der eigenen Fortpflanzung gehindert zu werden.« Gutmann 2005, 134. 
Bundesverfassungsgerichts (Hg.): Entscheidungen des Bundesverfassungsgerichts. Bd. 78. Tübingen 1989, 104-123.

Bundesverfassungsgericht: Beschluß vom 22. Juni 1988-2 BvR 234/87, 1154/86. In: Mitglieder des Bundesverfassungsgerichts (Hg.): Entscheidungen des Bundesverfassungsgerichts. Bd. 78. Tübingen 1989, 374-390.

Bundesverfassungsgericht: Urteil vom 28. Mai $1993-2$ BvF 2/90 und 4, 5/92. In: Mitglieder des Bundesverfassungsgerichts (Hg.): Entscheidungen des Bundesverfassungsgerichts. Bd. 88. Tübingen 1993, 203-366.

Bundesverfassungsgericht: Beschluß vom 28. Juni 1994 - BvL 14, 15/88. In: Mitglieder des Bundesverfassungsgerichts (Hg.): Entscheidungen des Bundesverfassungsgerichts. Bd. 91. Tübingen 1995, $118-124$.

Carraro, N.: Aristotle on Generation and Alteration. Dissertation LMU München 2014.

Damschen, G./Schönecker, D. (Hg.): Der moralische Status menschlicher Embryonen. Pro und contra Spezies-, Kontinuums-, Identitäts- und Potentialitätsargument. Berlin 2003.

Dietrich, F./Czerner, F.: Menschenwürde und vorgeburtliches Leben. In: Joerden, J./Hilgendorf, E./Thiele, F. (Hg.): Menschenwürde und Medizin. Ein interdisziplinäres Handbuch. Berlin 2013, $491-524$.

Embryonenschutzgesetz (ESchG).

Europäischer Gerichtshof (EuGH): Urteil vom 18.10.2011 - C34/10.

Gewirth, A.: Reason and Morality. Chicago 1978.

Gewirth, A.: Human Dignity as the Basis for Rights. In: Meyer, M. J./Parent, W. A. (Hg.): The Constitution of Rights. Human Dignity and American Values. Ithaca/London 1992, 10-28.

Graff, D./Williamson, T. (Hg.): Vagueness. Aldershot 2002.

Grice, H. P.: Studies in the Way of Words. Cambridge, MA/London 1989.

Grundgesetz (GG).

Günther, H.-L./Kaiser, P./Taupitz, J.: Embryonenschutzgesetz. Juristischer Kommentar mit medizinisch-naturwissenschaftlichen Einführungen. Stuttgart 2008.

Gutmann, T.: Rechtliche und rechtsphilosophische Fragen der Präimplantationsdiagnostik. In: Gethmann-Siefert, A./Huster, S. (Hg.): Recht und Ethik in der Präimplantationsdiagnostik. Bad Neuenahr-Ahrweiler 2005, $131-185$.

Huber, J.: Totipotenz - überfordertes Kriterium der Schutzwürdigkeit? Münster 2009. Hucklenbroich, P.: Individuation, Kontinuität und Potenzial. Zum Paradigmastreit in der Theorie der Reproduktion. In: Siep, L./Quante, M. (Hg.): Der Umgang mit dem beginnenden menschlichen Leben. Münster 2003, 37-57.

Keefe, R./Smith, P. (Hg.): Vagueness: A Reader. Cambridge 1997.

Keil, G.: Über die deskriptive Unerschöpflichkeit der Einzeldinge. In: Keil, G./Tietz, U. (Hg.), Phänomenologie und Sprachanalyse. Paderborn 2006, 83-125.

Keil, G.: Introduction: Vagueness and Ontology. In: Metaphysica 14/2, 2013, 149-164.

Kersten, J.: Das Klonen von Menschen. Eine verfassungs-, europa- und völkerrechtliche Kritik. Tübingen 2004.

Knoepffler, N.: Der moralische Status des frühen menschlichen Embryos. Nova Acta Leopoldina NF 96, 2007, Nr. 354, $177-188$.

Kongregation für die Glaubenslehre: Ecclesia Catholica, Sancta Sedes: Erklärung über den Schwangerschaftsabbruch. Acta Apostolicae Sedis (AAS) 66, 1974, 730-747.

Kongregation für die Glaubenslehre: Donum Vitae. Instruktion der Kongregation für die 
Glaubenslehre über die Achtung vor dem beginnenden menschlichen Leben und die Würde der Fortpflanzung. Verlautbarungen des Apostolischen Stuhls 74. Bonn 1987. Merkel, R.: Forschungsobjekt Embryo - Verfassungsrechtliche und ethische Grundlagen der Forschung an menschlichen embryonalen Stammzellen. München 2002.

Pardey, U.: Begriffskonflikte in Sprache, Logik, Metaphysik. Paderborn 2006.

Peirce, C. S.: Collected Papers. Vol. 1. Cambridge 1931.

Raffman, D.: Unruly Words. Oxford 2013.

Rager, G.: Menschsein zwischen Lebensanfang und Lebensende. Grundzüge einer medizinischen Anthropologie. In: ders. und Honnefelder, L. (Hg.): Ärztliches Urteilen und Handeln. Zur Grundlegung einer medizinischen Ethik. Frankfurt 1994, 53- 103.

Reich, J.: Empirische Totipotenz und metaphysische Gattungszugehörigkeit bei der moralischen Beurteilung vorgeburtlichen Lebens. In: Zeitschrift für medizinische Ethik 50/2, 2004, $115-130$.

Schöne, T.: Was Vagheit ist. Paderborn 2011.

Singer, P.: Praktische Ethik. 2., erw. Aufl. Stuttgart 1994.

Stammzellgesetz (StZG).

Stoecker, R.: Der Hirntod. 2. Aufl. Freiburg 2010.

Strafgesetzbuch (StGB).

Tertullian: Apologeticum. Übers. von Tobias Georges, Freiburg 2011.

Thomas von Aquin: Summa Theologica. Dt.-lat. Ausg., übers. von Dominikanern u. Benediktinern Deutschlands u. Österreichs. Graz u. a. $1933 \mathrm{ff}$.

Walter, S. (Hg.): Vagheit. Paderborn 2005.

Wiesemann, C.: Wie kann über den Embryo in einer lebensweltlich angemessenen Weise gesprochen werden? Eine Kritik der Debatte um den moralischen Status des Embryos. In: Graumann, S./Schneider, I. (Hg.): Verkörperte Technik - Entkörperte Frau. Biopolitik und Geschlecht. Frankfurt am Main 2003, 141-151.

Williamson, T.: Vagueness. London 1994. 